\title{
Study on the Influence of LED Spectrum on the Visual and Nonvisual Effects in the Interior Zone of Tunnels
}

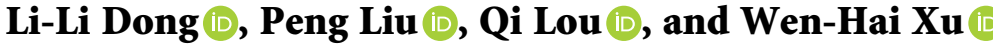 \\ School of Information Science and Technology, Dalian Maritime University, Dalian 116026, China \\ Correspondence should be addressed to Li-Li Dong; donglili@dlmu.edu.cn
}

Received 12 May 2021; Accepted 25 July 2021; Published 3 August 2021

Academic Editor: Elżbieta Macioszek

Copyright ( $) 2021 \mathrm{Li}-\mathrm{Li}$ Dong et al. This is an open access article distributed under the Creative Commons Attribution License, which permits unrestricted use, distribution, and reproduction in any medium, provided the original work is properly cited.

\begin{abstract}
Judicious use of lamps is of profound significance to improve the internal traffic safety of tunnels. This study evaluated the biological effects of spectrum on human visual and nonvisual under mesopic vision category. According to the difference of human eyes' response to the visual and nonvisual biological effects of different wavelengths of light radiation, a method of applying the mesopic visual spectral luminous efficiency curve to the assessment of nonvisual biological effects and blue light hazards is proposed. The results show that, in theory, under mesopic vision category, the spectrum with high correlation color temperature (CCT) and high color rendering index (CRI) has higher blue light content $Z$-value and has greater impact on human nonvisual effects and blue light radiation damage, but it can also provide higher human perception luminance. The visual effect detection experiment of driving in the interior zones of the tunnel is simulated in the laboratory, and the critical flicker frequency and reaction time are taken as physiological indexes. The results show that the spectrum with high CCT and high CRI corresponds to lower critical flicker frequency drop value and faster reaction time, which has a positive effect on improving driving safety. Therefore, this paper suggests that the LED with high CCT and high CRI (CCT $=6500 \mathrm{~K}, \mathrm{CRI} 2012=95)$ should be used in the interior zones of the tunnel with relatively short lighting section, and the CCT and CRI should be appropriately reduced in other road environments with long lighting section to reduce the impact of spectrum on nonvisual photobiology, so as to improve road traffic safety.
\end{abstract}

\section{Introduction}

Fatigue and drowsiness are the main causes of traffic accidents [1-3]. Due to the semienclosed space characteristics of highway tunnels, its visual pressure and monotonous environment are more likely to aggravate the fatigue and irritability of drivers, resulting in decreased driver alertness, inattention, and performance [4-6]. At the same time, the impact of tunnel internal accidents is usually greater than that of open sections owing to the closed characteristics $[7,8]$. The lighting environment directly affects the driver's information cognition and then affects the driving safety. There is no natural light to provide lighting in the tunnel. The driver can only obtain the visual capture information through the artificial light source [9]. Therefore, it is imperative to research tunnel lighting from the perspective of traffic safety.
The traditional tunnel lighting design only pays attention to whether the driver can identify the obstacles and does not consider the influence of the light source on human physiology and psychology. However, studies have shown that light not only provides us with visual information, but also produces nonvisual biological effects that affect many physiological and behavioral processes such as emotion and activity [10]. In particular, CIE "position statement on nonvisual effects of light" on Oct. 3, 2019, clearly stated that "lighting should consider the visual and nonvisual effects of light comprehensively." Therefore, in the tunnel interior lighting settings, we should fully consider the impact of light on human visual and nonvisual biological effects, to ensure driving safety as far as possible. According to the current tunnel lighting standards, the recommended luminance values for different tunnel areas are given [11]. However, the lack of guidance on the selection of correlated color 
temperature (CCT) and color rendering index (CRI) of tunnel lamps has led to random settings of CCT and CRI of tunnel lighting, which has a negative impact on the safety of tunnel operations. Therefore, from the perspective of spectrum, this paper studied the effects of CCT and CRI on human visual and nonvisual biological effects under mesopic vision category.

After decades of in-depth research, the influence of spectrum on visual and nonvisual biological effects has been proved to exist $[12,13]$. In the current research, people think that the nonvisual effect is mainly related to retinal ganglion cells (ipRGCs) containing melanin protease [14]. However, existing studies have shown that rods and cones also affect ipRGCs. The proportion of these three kinds of cells in biological effects is not clear, but it is certain that their proportion will change with the change of light intensity [15]. At the same time, their spectral sensitivity is different, which means that under different brightness, the influence of spectrum on visual and nonvisual effects is different. Christian et al. found that the alert response to light is related to the wavelength. Shortwavelength light $(460 \mathrm{~nm})$ is more efficient than long-wavelength light $(550 \mathrm{~nm})$ to reduce sleepiness at night [16]. PhippsNelson et al. found that blue light exposure at night can reduce drowsiness [17]. Sahin and Figueiro have shown that red light can improve human alertness more than blue light in the afternoon [18]. Lisdiani and Yassierli studied the effect of blue light on human alertness at night and found that adding the blue light to the light environment can improve the alertness of office workers at night [19]. Figueiro et al. found that exposure to red light not only increased objective alertness but also significantly reduced the reaction time (i.e., improved performance) when performing GO/NOGO tasks at night [20].

At present, the study of the influence of the spectrum on human photobiological effect is usually conducted under photopic vision category. However, existing research shows that for human, even if exposed to low-intensity light, many physiological processes of human will be affected. Vision is a complex process of perception and thinking. Human eyes change with the change of light brightness in the visual environment. Therefore, it is unreasonable to apply the conclusion under photopic vision category to the mesopic vision category such as road lighting. Decision makers and road lighting industry need to evaluate the impact of light source on the biological effects of visual and nonvisual under mesopic vision category.

The purpose of this study is to study the influence of spectral components on people from the perspective of visual effect and nonvisual effect under mesopic vision category and to find more suitable CCT and CRI parameters for tunnel lighting environment, improve the quality of tunnel lighting, and improve the safety of road lighting. These conclusions are of great significance to improve road safety and provide reference for tunnel traffic safety lighting design.

\section{Materials and Methods}

Based on the complexity of nonvisual biological effects and the particularity of tunnel lighting, this paper first selects the color coordinate $Z$-value of $1931 \mathrm{CIE}-X Y Z$ standard chroma system, circadian rhythm factor (CAF), perception luminance, and blue light hazard factor to evaluate the influence of spectrum on visual and nonvisual biological effects. The theoretical results show that the spectra with high CCT and CRI correspond to higher $Z$-value, CAF-value, perceived brightness, and blue light hazard factor.

2.1. LED Spectrum Used in Road Lighting. At present, tunnel lighting mainly uses the blue light to excite the yellow phosphor to make the light-emitting diode (LED), whose spectrum presents the double peak characteristic. The CCT and CRI of the light source are determined by the spectral content at different wavelengths. In the experiment, seven kinds of CCT are selected, which are $3000 \mathrm{~K}, 3500 \mathrm{~K}, 4000 \mathrm{~K}$, $4500 \mathrm{~K}, 5000 \mathrm{~K}, 5700 \mathrm{~K}$, and $6500 \mathrm{~K}$. Our previous research shows that CRI2012 has a good evaluation effect on tunnel lighting environment from several current color rendering evaluation indexes [21]. Therefore, this paper uses CRI2012 to study the CRI of tunnel led. The brightness of the light source used in the tunnel is generally between 60 and 90 . In order to analyze the spectra of different CRIs, four CRIs (65, 75, 85, and 95, resp.) were selected. SPDs of different CCTs and CRIs are shown in Figure 1 and measured using a I1Pro spectroradiometer.

Figure 1(a) shows the spectra of seven CCTs with a CRI of 85, and Figure 1(b) shows the spectra of four CRIs with a CCT of $3000 \mathrm{~K}$. Other spectra have the same trend. In the theoretical calculation and practical experiment, the spectrum value measured under the same tunnel illumination brightness is used.

2.2. Blue Light Content. The blue wavelength part of the light source is not the main sensitive band of human eye sensitivity, which has little effect on improving the efficiency of the light source, but plays a certain role in the CCT and CRI, so it is an indispensable wavelength for the light source with good light color performance. In recent years, relevant theoretical studies have shown that the intermediate vision theory, nonvisual light biological effect and blue light damage are directly related to the blue light wavelength content, or even play a major role. CIE-XYZ system was defined by CIE in 1931 to determine the three primary colors (red, green, and blue) in the spectrum and then to represent all colors in the spectrum on a two-dimensional plane. $Y$ and $Z$ represent the relative values related to red, green, and blue, respectively, and $Z=1-(X+Y)$. In this paper, the chromaticity coordinate $Z$-value is used to evaluate the blue light content in the spectrum. Figure 2 shows the chromaticity coordinate $Z$-value of the spectrum shown in Figure 1.

The $Z$-value in Figure 2 represents the content of blue light components corresponding to different spectra. Fitted is to use the fit linear function of Origin software to obtain the relationship between $Z$ and CCT by linear fitting with least square method. $R^{2}$ represents the square of correlation coefficient. It can be seen from Figure 2 that for the LED spectrum now used, the blue light content of the spectrum shows an obvious linear increasing trend with the increase of CCT and CRI. 


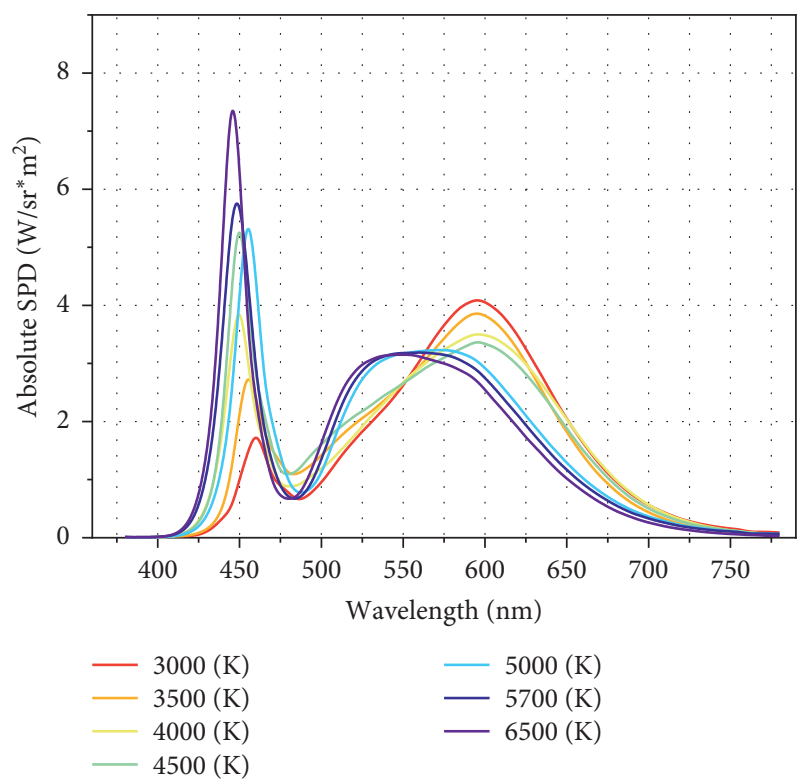

(a)

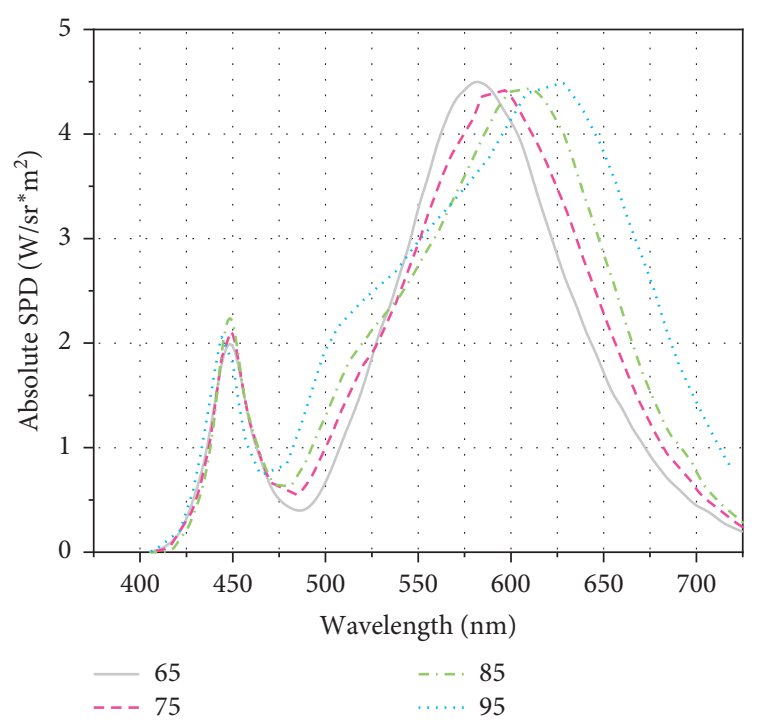

(b)

Figure 1: Absolute spectral power distributions. (a) CRI2012 $=80$. (b) CCT $=3000$.

2.3. Circadian Action Factor. In recent years, CAF is regularly used to quantitatively reflect the influence of the spectrum on human nonvisual biological effects [15, 22-24]. In workplaces, lights with high CAF values can enhance workers' level of vigilance and excitation, while in bedrooms, the light with low CAF values are required to help people relax $[23,25,26]$.

$$
\mathrm{CAF}=\frac{K_{c} \int_{380}^{780} L_{e d}(\lambda) * C(\lambda) \mathrm{d} \lambda}{K_{e} \int_{380}^{780} L_{e d}(\lambda) * E(\lambda) \mathrm{d} \lambda},
$$

where $L_{e d}(\lambda)$ denotes the SPD of the light source. $E(\lambda)$ denotes the visual spectral luminous efficiency function. $C$ $(\lambda)$ denotes the spectral circadian efficiency function. $K_{c}$ denotes the maximum spectral luminous efficiency.

$E(\lambda)$ refers to the spectral luminous efficiency function of the human eye. Photoreceptor cells in the retina can be divided into two types: cones and rods. The luminance of tunnel lighting belongs to the range of mesopic vision. At this time, the rod cells and cone cells work at the same time and the specific gravity changes with the luminance. At the same time, due to the different spectral sensitivity curves of the two kinds of cells, the spectral luminous efficiency function also changes with the luminance. Our previous research shows that it is reasonable to use the MES-2 model to calculate the mesopic visual curve in the tunnel lighting area [27]. Therefore, in order to better analyze the nonvisual biological effects of different spectra under tunnel illumination, we use the MES-2 model to calculate the mesopic visual curve corresponding to different spectra and take it as $E(\lambda)$. The form of the MES-2 model is as follows:

$$
\begin{aligned}
L_{s} & =K_{m}^{\prime} \int_{380}^{780} V^{\prime}(\lambda) L_{e d}(\lambda) \mathrm{d} \lambda, \\
L_{p} & =K_{m} \int_{380}^{780} V(\lambda) L_{e d}(\lambda) \mathrm{d} \lambda, \\
L_{\text {mes, } n} & =\frac{m_{2,(n-1)} L_{p} K_{m}^{\prime}+\left(1-m_{2,(n-1)}\right) L_{s} K_{m}}{m_{2,(n-1)} K_{m}^{\prime}+\left(1-m_{2,(n-1)}\right) K_{m}}, \\
m_{2,0} & =0.5, \\
m_{2, n} & =0.3334 \log L_{\text {mes }, n}+0.767, \quad 0 \leq m_{2, n} \leq 1, \\
V_{\text {mes }}(\lambda) & =\frac{m_{2, \text { end }} V(\lambda)+\left(1-m_{2, \text { end }}\right) V^{\prime}(\lambda)}{\left(m_{2, \text { end }} / K_{m}\right)+\left(\left(1-m_{2, \text { end }}\right) / K_{m}^{\prime}\right)},
\end{aligned}
$$

where $V^{\prime}(\lambda)$ is the scotopic spectral luminous efficiency function, and $K_{m}^{\prime}$ is its peak value. $V(\lambda)$ is the photopic spectral luminous efficiency function, and $K_{m}$ is its peak value. $L_{e d}(\lambda)$ is the SPD of the light source. $L_{s}$ is the scotopic luminance. $L_{p}$ is the photopic luminance. Equations (4) and (5) are iterative processes, $n$ is an iteration step, and $m_{2, \text { end }}$ is the final iteration output. $V_{\text {mes }}(\lambda)$ is the mesopic spectral luminous efficiency function. Substituting the above $L_{e d}(\lambda)$ spectral curve into the formula calculation, the mesopic spectral luminous efficiency curve corresponding to each spectrum is shown in Figure 3.

Figure 3 shows the mesopic spectral luminous efficiency curve corresponding to the spectrum. It can be seen from Figure 3 that the difference is slight between mesopic 


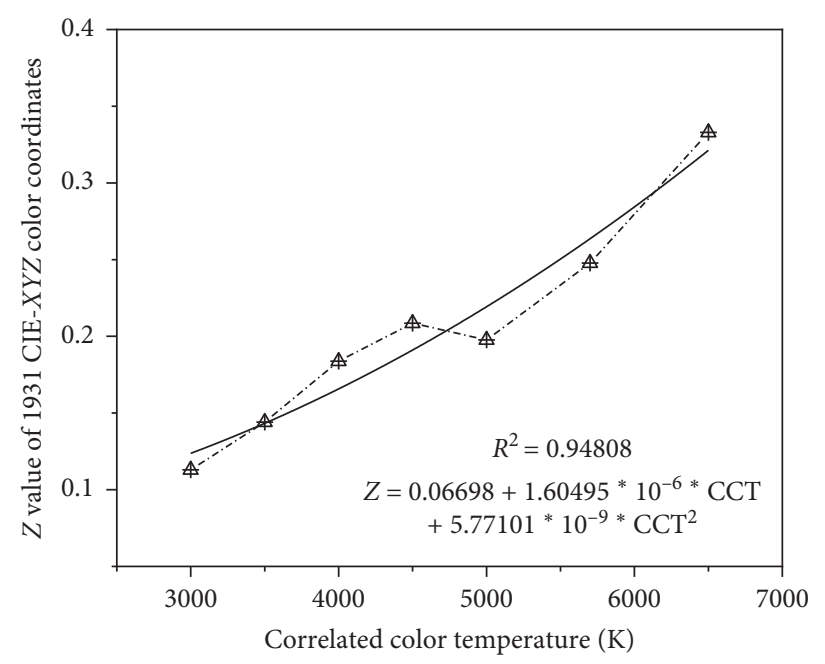

- $4-Z$ value

— Fitted

(a)

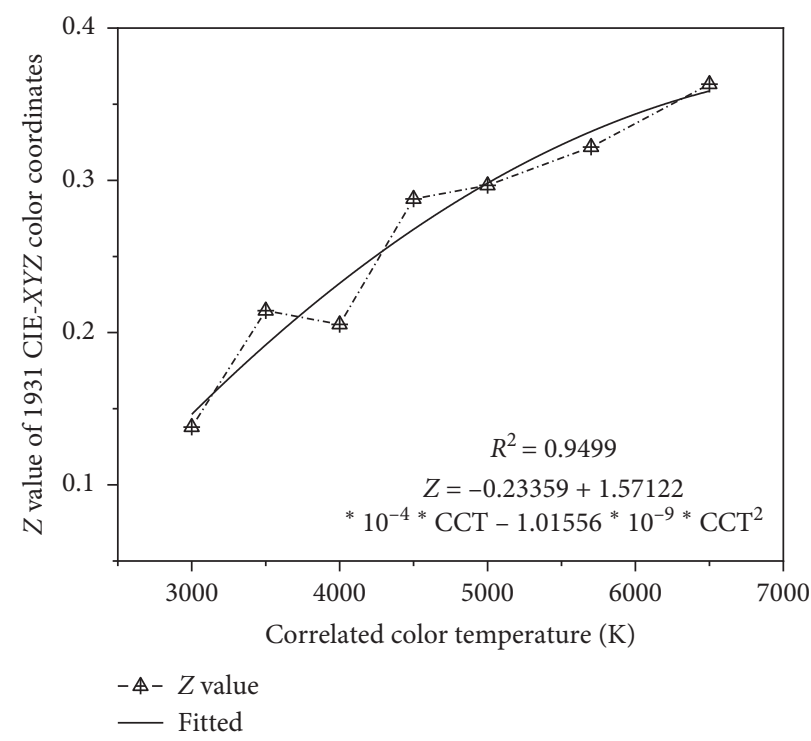

(c)

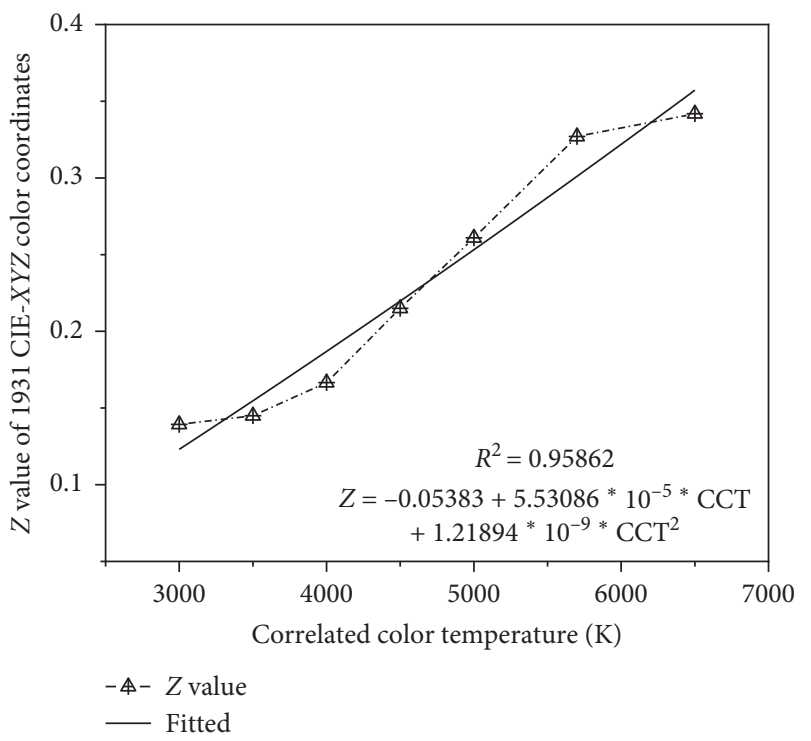

(b)

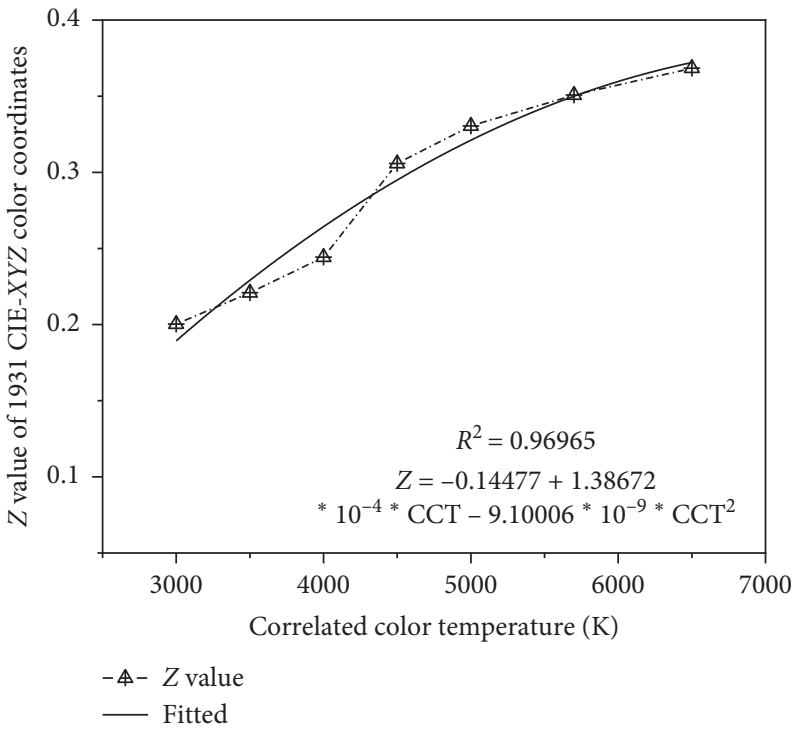

(d)

Figure 2: Z-value of 1931 CIE-XYZ color coordinates. (a) CRI2012=65. (b) CRI2012=75. (c) CRI2012=85. (d) CRI2012=95.

spectral luminous efficiency functions under different CCTs. The low CCT and low CRI spectrum correspond to a higher mesopic spectral luminous efficiency curve.

At present, $C(\lambda)$ is mainly drawn by the influence of different wavelengths of light on a certain physiological parameter of the human body. There are four main $C(\lambda)$ models: Brainard model based on melatonin inhibition, Thapan model based on melatonin inhibition, Jiaqi Ju model based on heart rate change, and Berman model based on pupil size change [22, 28-30]. Recent studies have shown that the nonvisual biological effect is mainly caused by the action of ipRGCs, which can transmit the induced light signal to the supraoptic nucleus and then control the secretion of melatonin in pineal gland [21, 31-34]. Melatonin can increase fatigue and accelerate the state of sleepiness, which leads to poor reaction ability and judgment ability and affects automatic function [35]. The nonvisual bioeffect evaluation curve based on melatonin inhibition is shown in Figure 4. According to the experimental results of Brainard and Thapan on the spectrum of photoinduced melatonin inhibiting several wavelengths, Gall defines a circadian rhythm action function $C(\lambda)$ [36].

The CAF values corresponding to different spectra are obtained by substituting $E(\lambda)$ and $C(\lambda)$ into the circadian rhythm factor calculation formula, as shown in Figure 5.

CAF values in Figure 5 represent the values of circadian rhythm factors corresponding to different spectra. It can be seen from Figure 5 that the circadian rhythm factors of LED with different spectra show an obvious linear increasing trend with the increase of color temperature and color 


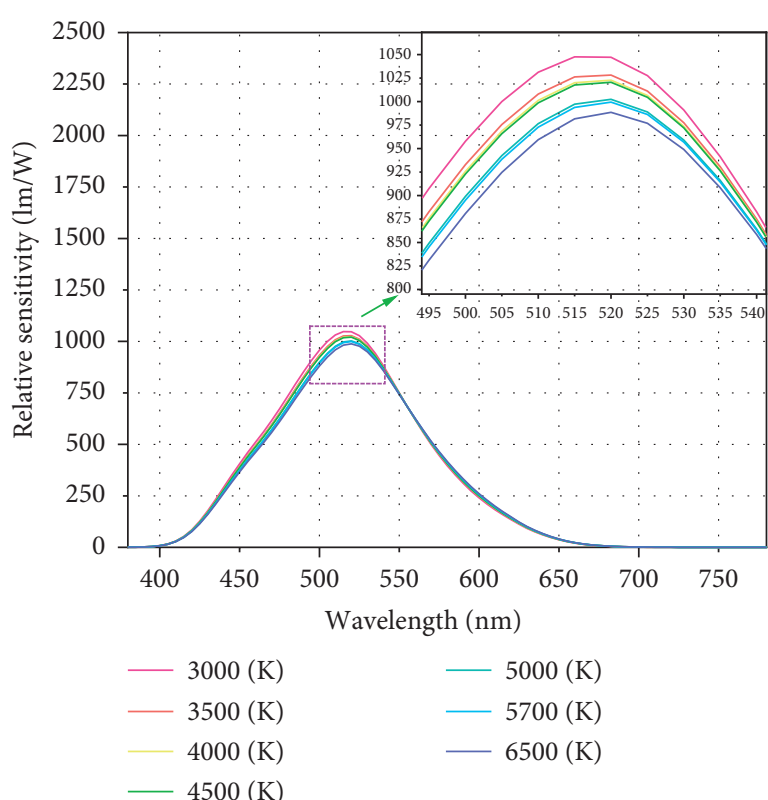

(a)

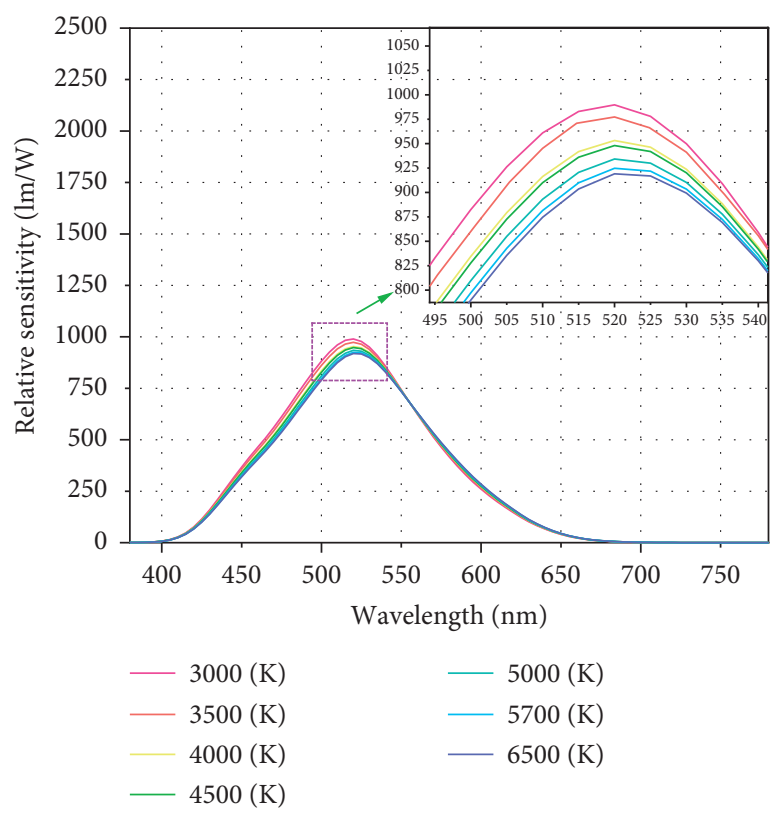

(c)

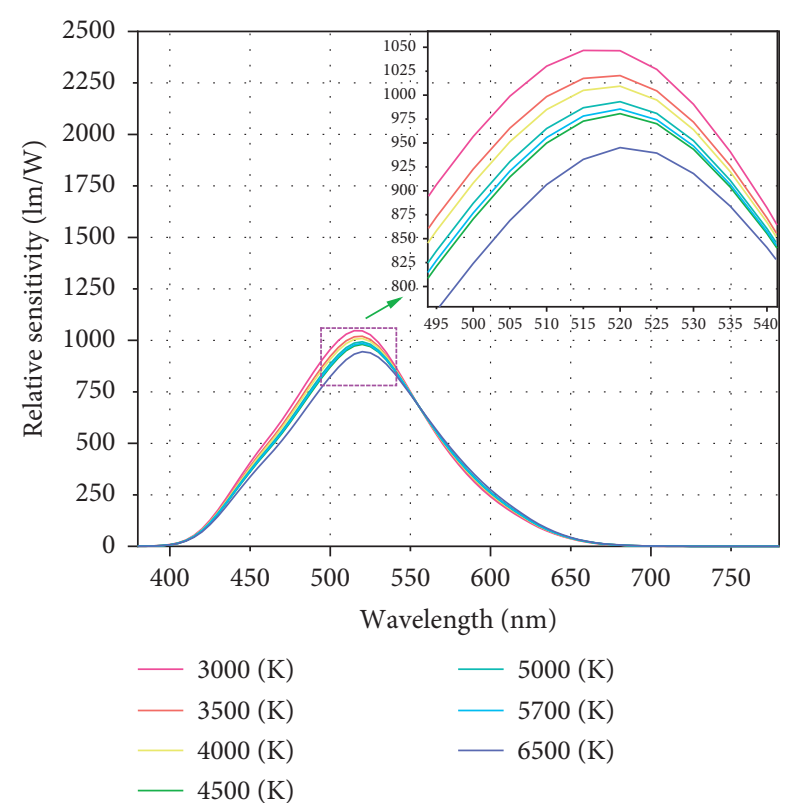

(b)

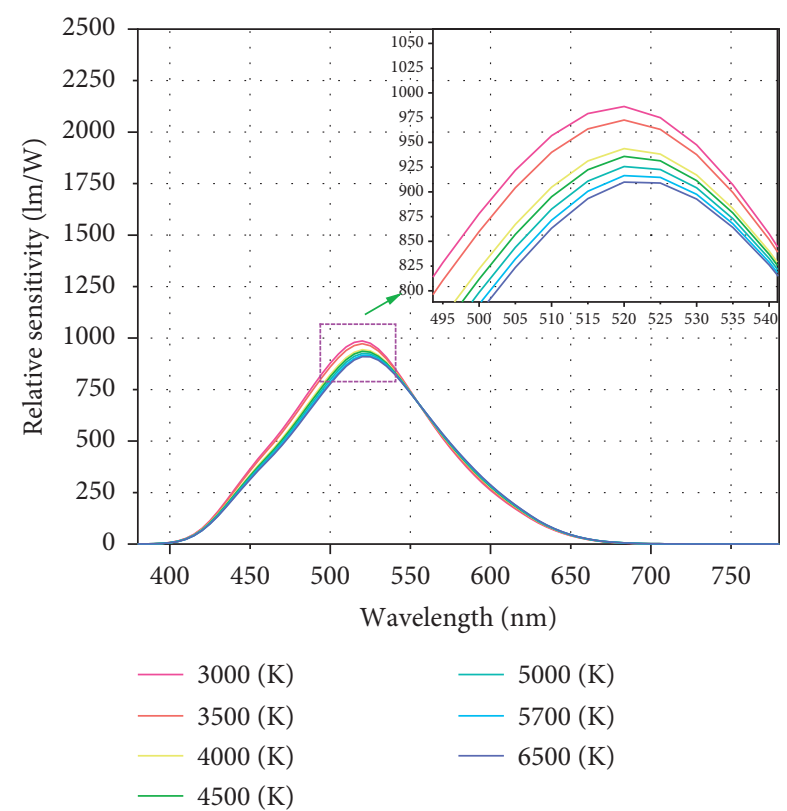

(d)

Figure 3: Mesopic spectral luminous efficiency. (a) CRI2012 =65. (b) CRI2012=75. (c) CRI2012=85. (d) CRI2012=95.

rendering. That is, with the increase of color temperature and color rendering, the circadian rhythm factor corresponding to the spectrum increases, and the nonvisual biological effect increases.

2.4. Perception Luminance. With the development of lighting technology, the composition of light environment is becoming more and more diversified. It is very meaningful to study the perception of human eyes to the light environment, especially for the environment with low brightness such as road lighting. Traditional luminance meter is only used to measure the brightness of the object under test, and it is an objective physical quantity, not the brightness that the human eye can actually perceive. In order to better analyze the applicability of different spectra in road lighting environment, the human eye perception luminance corresponding to different spectra in the mesopic vision is calculated. The calculation formula of human eye perceived luminance under mesopic vision is as follows:

$$
L_{\mathrm{pl}}=L_{b} \int_{380}^{780} L_{e d}(\lambda) V_{\mathrm{mes}}(\lambda) \mathrm{d} \lambda,
$$

where $L_{\mathrm{pl}}$ is the perceived luminance of the human eye under mesopic vision, $L_{b}$ is the brightness of the light environment, 


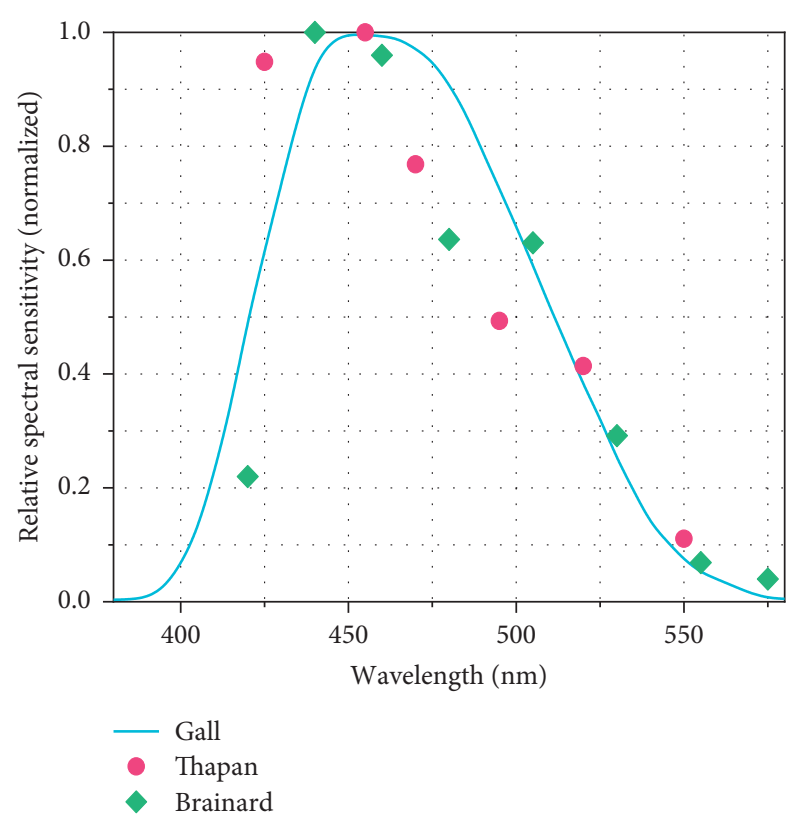

Figure 4: Nonvisual biological effects model.

and $V_{\text {mes }}(\lambda)$ is the mesopic visual curve shown in Figure 3. The results of normalization are shown in Figure 6.

The results show that the white LED spectrum with high CCT and CRI can provide higher human eye perception luminance.

2.5. Blue Light Hazard. Due to the production process, production cost, and other factors, most of the road lighting uses blue light to excite yellow phosphor to produce white light. As blue light is the main light-emitting device, the blue spectrum component of white LED spectrum accounts for a large proportion, thus forming the blue light problem. And more and more electrical designers and ordinary consumers know and concern. There is no requirement for blue light hazard index in current main road lighting design specifications and standards. The blue light harm of LED lamps is controllable. Only by correctly understanding and fully considering the blue light harm of LED lamps can the blue light harm be avoided correctly and reasonably. In the current research, the calculation formula of the blue light damage efficiency $K_{b}$ of light radiation is as follows:

$$
K_{b}=\frac{\int_{380}^{780} L_{e d}(\lambda) * B(\lambda) \mathrm{d} \lambda}{K_{e} \int_{380}^{780} L_{e d}(\lambda) * E(\lambda) \mathrm{d} \lambda},
$$

where $L_{e d}(\lambda)$ represents the SPD of the light source, $B(\lambda)$ represents the spectral weighting function of retinal blue light damage, $E(\lambda)$ represents the spectral luminous efficiency function of human vision, and $K_{e}$ is its maximum spectral luminous efficiency. By analogy with the above circadian rhythm factor calculation process, we still use the mesopic visual spectral luminous efficiency curve in Figure 3 as $E(\lambda)$. Figure 7 shows the spectral weighting function of retinal blue light damage $B(\lambda)$.
Substituting the LED spectrum into the above blue light hazard efficiency calculation formula, the blue light hazard factors corresponding to different spectra are obtained, as shown in Figure 8.

From the fitting results in Figure 8, it can be seen that the blue light hazard factors of LED with different spectra show an obvious linear increasing trend with the increase of CCT and CRI. It means that with the increase of CCT and CRI, the damage of blue light caused by the spectrum is more serious.

\section{Experiment}

3.1. Selection of Experimental Indicators. According to the above theoretical analysis, we come to the conclusion: in the intermediate vision, high CCT and high CRI spectra with high blue light components can provide higher perceived luminance for human eyes, but will have a greater impact on human nonvisual photobiological effects, and the potential blue light harm is also higher. In the actual tunnel driving environment, the influence of different spectra on human visual efficacy needs to be further studied. Therefore, we select the critical flicker frequency (CFF) [37-40] and reaction time as the characterization parameters of visual experiment and analyze the changes of CFF and reaction time under different light environments through relevant experiments, so as to realize the evaluation of driver's visual performance under different spectra.

As an organ that directly feels light stimulation, the change of eye activity can most directly reflect visual fatigue. The research of Iwasaki and Akiya confirmed that the decline of CFF reflects the weakening of retinal function and measures the fatigue degree of human eyes. As a reflection of high-level neural activity and physiological state, it has been widely used to detect the activity level and arousal level of cerebral cortex in medicine in recent years [41-43]. With the increase of flash frequency, the phenomenon that human eyes regard flash light as continuous light is called flash fusion, and the minimum stimulus frequency causing fusion is called CFF. The reason for this phenomenon is that after the light stimulation stopped, the nerve excitation caused by it did not disappear immediately, but left a certain trace in the cerebral cortex, so the visual image did not disappear immediately, but stayed for a period of time, resulting in visual residue. Visual traces give us the illusion that light is continuous. The lower the CFF value, the greater the fatigue degree [44]. Compared with other indexes, CFF is simple and accurate in measuring visual fatigue.

Detection methods based on stimulus response ability are often used to evaluate driving behavior [45-48]. The process of human visual response includes five stages: investigation, discovery, recognition, recognition, and decision-making. Reaction time is the time from the beginning of stimulation to the beginning of observer reaction. At the same time, reaction time reflects the comprehensive influence of visual psychology and physiology on driving visual behavior in the process of road lighting, and is an important evaluation index for early recognition of obstacles and dangerous situations [49]. 


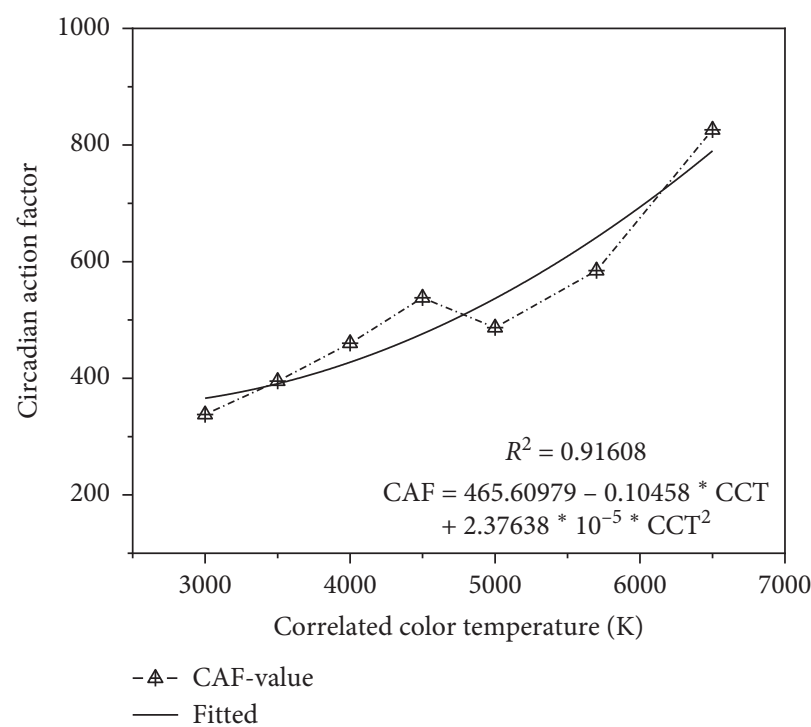

(a)

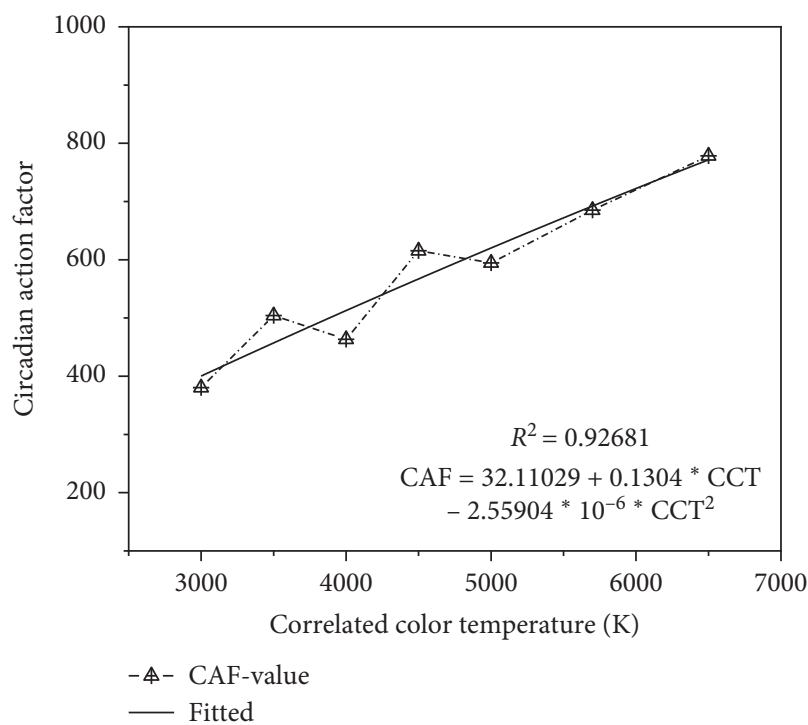

(c)

FIgURE 5: Circadian action factor. (a) CRI2012 $=65$.

The main purpose of this experiment is to test the effect of CCT and CRI on human eye performance in tunnel driving environment.

3.2. Parameter Settings. In order to study the effect of CCT and CRI on reaction time and CFF, three experimental parameters were set up, including CCT, CRI, and target. Seven kinds of CCTs, four kinds of CRIs, and nine kinds of target sizes were selected in this paper.

Spectra corresponding to seven CCTs $(3000 \mathrm{~K}, 3500 \mathrm{~K}$, $4000 \mathrm{~K}, 4500 \mathrm{~K}, 5000 \mathrm{~K}, 5700 \mathrm{~K}$, and $6500 \mathrm{~K}$ ) and four CRIs $(65,75,85$, and 95$)$ were selected to study the influence of spectra on visual efficiency. Three kinds of mesopic visual luminance are set: $1 \mathrm{~cd} / \mathrm{m}^{2}, 2 \mathrm{~cd} / \mathrm{m}^{2}$, and $3 \mathrm{~cd} / \mathrm{m}^{2}$, which is consistent with the actual luminance range of the tunnel.

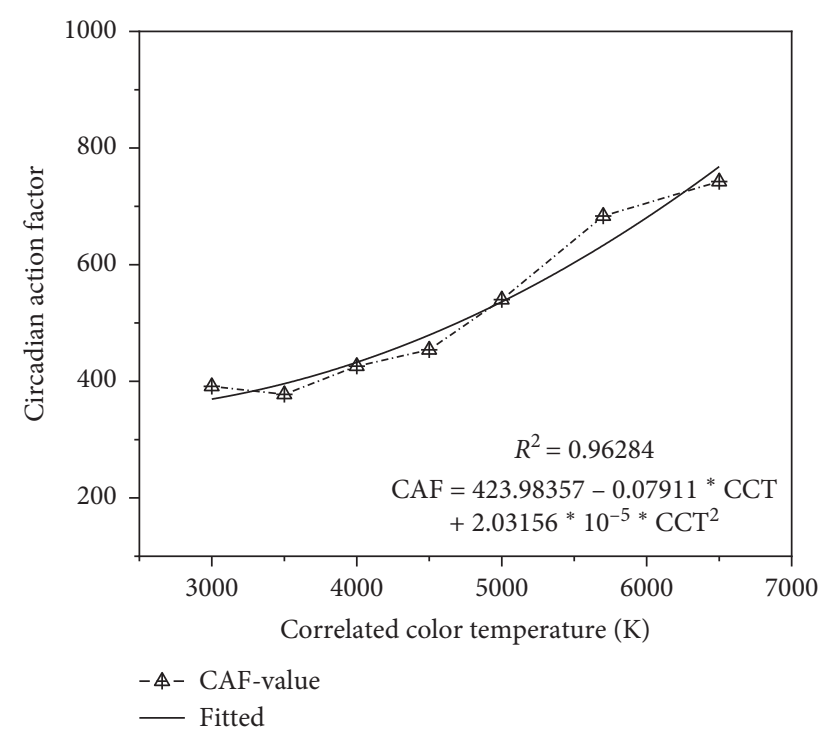

(b)

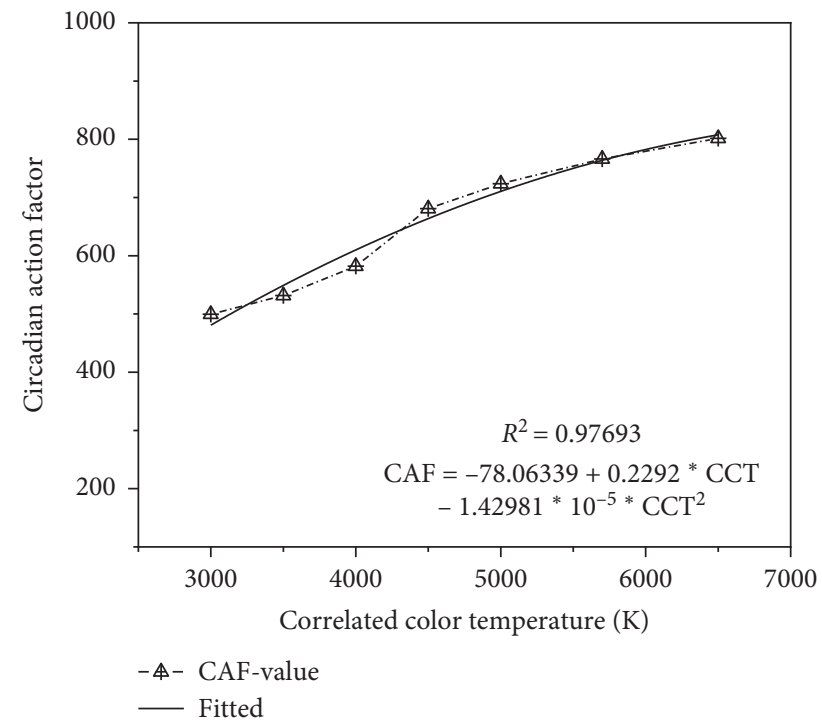

(d)

(b) CRI2012 $=75$. (c) CRI2012 $=85$. (d) CRI2012 $=95$.

In the experiment, the red Landolt loop is used as the target. Our previous experiments found that the red target can be detected quickly, but its gap direction is relatively difficult to distinguish. Before the experiment, we set up nine groups of Landolt loop of different sizes to make sure that everyone could see the orientation of the targets, and chose the size as small as possible. The target is shown in Figure 9.

We set the target luminance according to the current road lighting requirements. The target contrast can be calculated by (7):

$$
C=\frac{L_{t}-L_{b}}{L_{b}},
$$

where $C$ is the target-background contrast, $L_{t}$ is the target luminance, and $L_{b}$ is the background luminance. In general, the standard of road lighting has a contrast of 0.4. Therefore, 


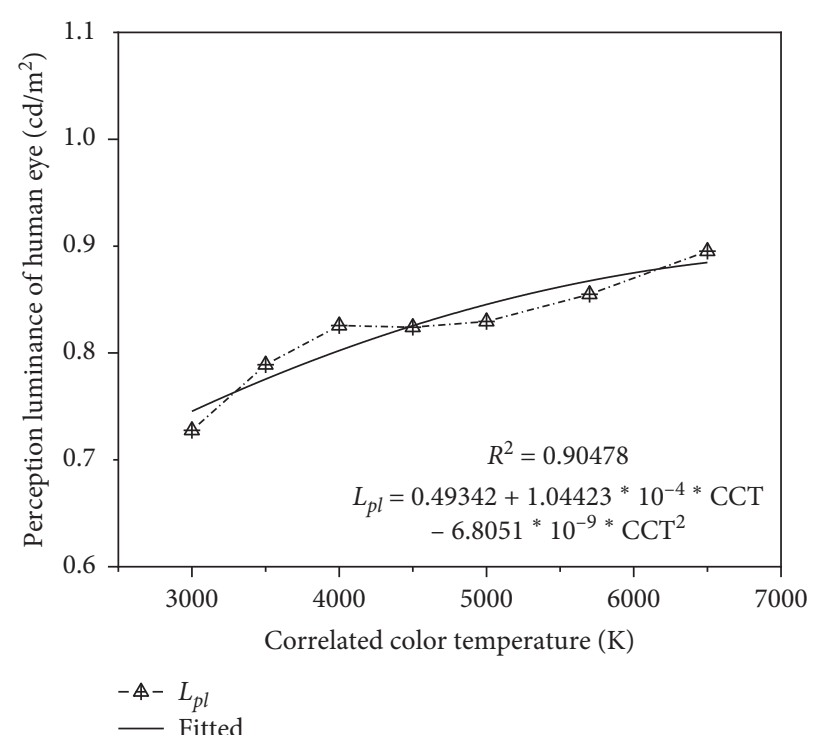

(a)

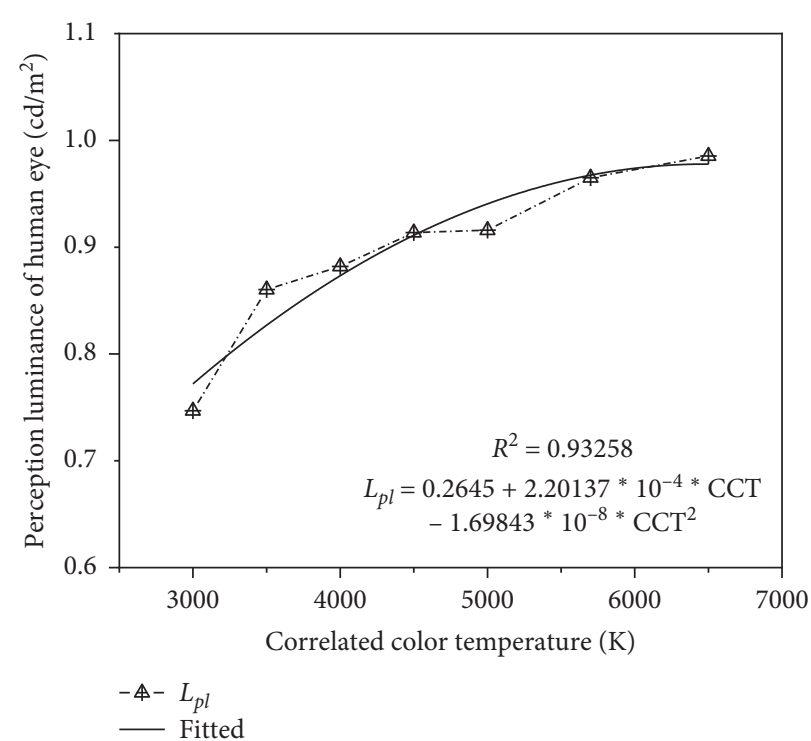

(c)

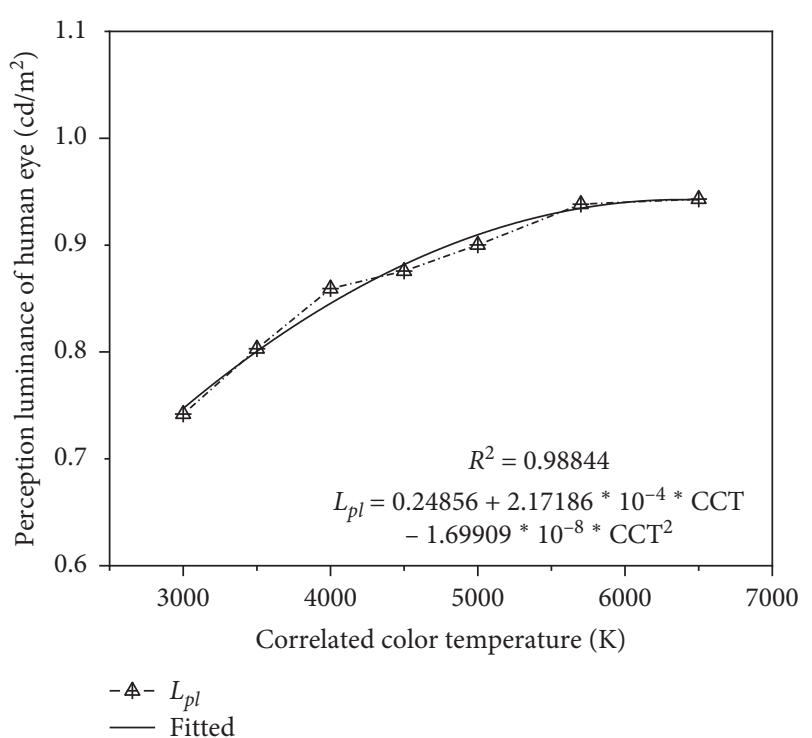

(b)

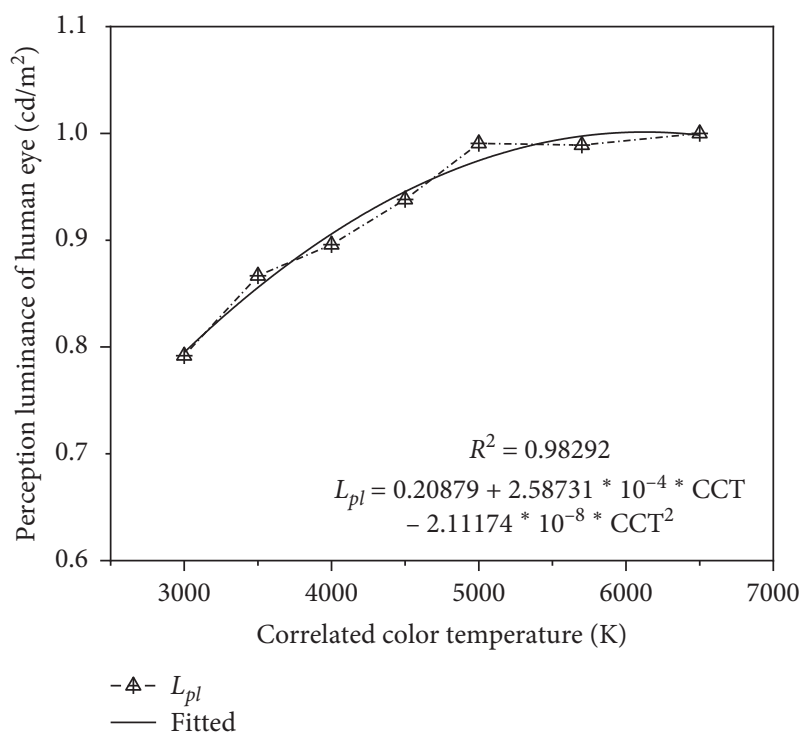

(d)

FIgure 6: Perception luminance of the human eye. (a) CRI2012 $=65$. (b) CRI2012 $=75$. (c) CRI2012 $=85$. (d) CRI2012=95.

we set three groups of target luminance corresponding to three kinds of background luminance. Table 1 shows the target luminance. In practice, we use laptops to display target. The target luminance is determined by changing the screen luminance of the laptop and detecting whether the luminance value of the Landolt loop meets the requirements by Konica Minolta CS-2000.

3.3. Experimental Setup. According to the principle of tunnel driving, a tunnel model with a width of $5 \mathrm{~m}$, a height of $3 \mathrm{~m}$, and a length of $10 \mathrm{~m}$ was built. In order to reduce the interference of external light as much as possible, an experimental platform was built to simulate the tunnel environment composed of two $2 \mathrm{~m} * 3 \mathrm{~m}$ and one $3 \mathrm{~m} * 3 \mathrm{~m}$ experimental plates, and a layer of wallpaper imitating the tunnel pavement and wallpaper were pasted on its surface. Figure 10 shows the real experimental situation corresponding to the seven CCTs. Figure 11 illustrates a schematic diagram of the experimental setup.

As shown in Figure 11, the observer is the experimental object. The luminaire's arrangement in the experiment adopts the mid-line symmetry method, and the angle is $36^{\circ}$, which conforms to the principle of luminaire arrangement and the requirement of the illumination angle in the tunnel. Flicker fusion apparatus is a bright spot scintillator named EP-403 produced by Beijing Lange Technology Company, which is used for CFF measurement. The adjustment range of flicker frequency is $8 \mathrm{~Hz} \sim 60 \mathrm{~Hz}$, and the display accuracy is less than $0.1 \mathrm{~Hz}$. Lenovo X395 is a laptop for displaying 


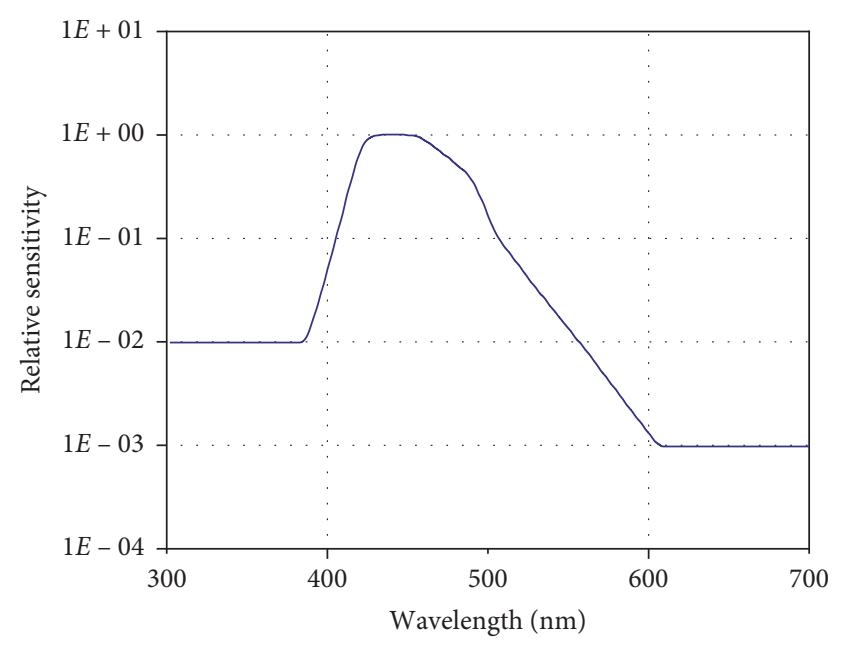

$-B(\lambda)$

Figure 7: The spectral weight function $B(\lambda)$ of blue light hazard on the retina.

targets. Bluetooth keyboard and laptop work together as a timing tool for subjects to respond to targets. PC is the computer that the experimenter uses to record the reaction time and CFF. The wallboard is a light barrier.

3.4. Procedure Specification. Forty subjects with normal vision (or corrected vision) with driver's licenses participated in the study, including 20 women and 20 men. The subjects were healthy and drug free. They all had normal color vision in terms of the Ishihara test. All had regular sleep-wake cycles with no complaints of sleep disorders (Pittsburgh sleep quality index $<5$ ). All subjects gave written informed consent.

In order to avoid mutual interference, CFF and reaction time experiments were carried out, respectively. For at least three nights prior to the study, subjects were required to keep a regular sleep-wake cycle (sleeping from 23.00 to $07.00 \mathrm{~h}$ ). For $24 \mathrm{~h}$ preceding each study session and for its duration, subjects refrained from caffeinated drinks, alcohol, excessive exercise, and bright light. The subjects participated in the experiment within a week, and they were asked to keep their daily life as consistent as possible for a week. The experimental process is as follows.

Firstly, the experiment adjusts the LEDCube to the light environment required. It should be noted that all light sequences in this experiment are random. Subjects were asked to remain seated and avoid head movement. They were instructed to keep their eyes open and focus their eyes on the center of the screen.

Secondly, the subjects were asked to measure CFF at the beginning of the CFF experiment. The frequency of CFF measurement was from low to high once and from high to low again. The CFF value recorded by the experimenter was the average value of the two measurements. CFF values were recorded at 5,10 , and 15 minutes using the same method. These times also correspond to the actual travel time in the tunnel.
Thirdly, the subjects were told to rest. After 5 minutes, the subjects repeated the experiment until all the light environment experiments were completed or the experiment time was over.

There are some differences between the CFF experiment and the reaction time experiment. We have made 30 groups of videos in advance, and a visual target will appear at 5, 10, and 15 minutes of the video. In order to avoid the subjects recording the direction of the visual target, the direction of the visual target in any video and at any time is set to be different, and the screen is in the black state for the rest of the time to reduce the interference to the experiment. The subjects had 10 seconds to distinguish the direction of the visual target after the appearance of the visual target, and the visual target would disappear after 10 seconds. After the visual target appears, the PC will start timing until the subject presses the corresponding position of the Bluetooth keyboard to finish timing. The subjects were told to identify the correct direction of the target as much as possible and then press the keyboard, and the wrong data will be discarded.

\section{Results and Discussion}

In order to familiarize the subjects with the whole experimental process, three preliminary experiments were being conducted in a random lighting environment before the formal experiment begins, and those results are not be considered in the final data. Through the experimental program, the CFF and reaction time values of 40 subjects under different light conditions were obtained.

4.1. Experimental Results and Discussion of CFF. In the experiment, $0 \mathrm{~min}$ of each experiment was used as the initial value, and the initial value minus the $C F F$ value at $5 \mathrm{~min}$, $10 \mathrm{~min}$, and $15 \mathrm{~min}$ as the CFF drop value. Higher CFF drop value suggests higher fatigue. The average CFF drop value with time in 40 subjects under different light environments is shown in Figure 12.

Figure 12 shows the average CFF drop value over time for all observers in different light environments. It can be seen from Figure 12 that the CFF drop value increases with time, which indicates that the fatigue of the experimenter is increasing with time. When the luminance and CRI were fixed, the CFF drop value decreases with the increase of CCT. When the luminance and CCT were fixed, the CFF drop value decreases with the increase of CRI. When the CCT and CRI were fixed, the decrease of CFF decreases with the increase of luminance. This shows that improving the CCT and CRI of tunnel lighting has a positive effect on improving the driver's visual performance.

In order to study the correlation between characteristic variables (CCT, CRI, luminance, and experimental duration) and target variables (CFF drop value and reaction time). The correlation coefficient and $P$ value were obtained by Pearson's correlation analysis of experimental data by SPSS software. 


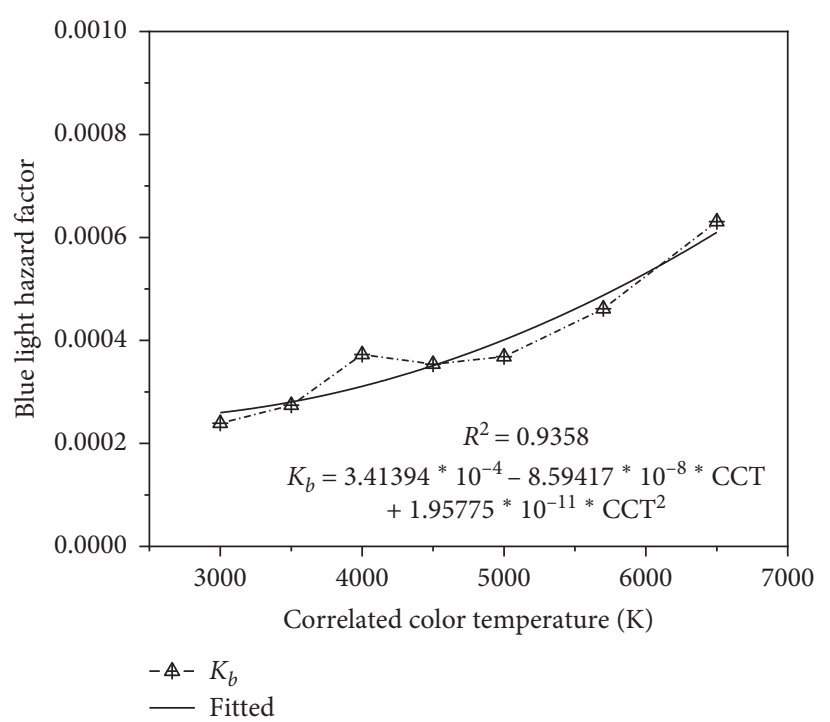

(a)

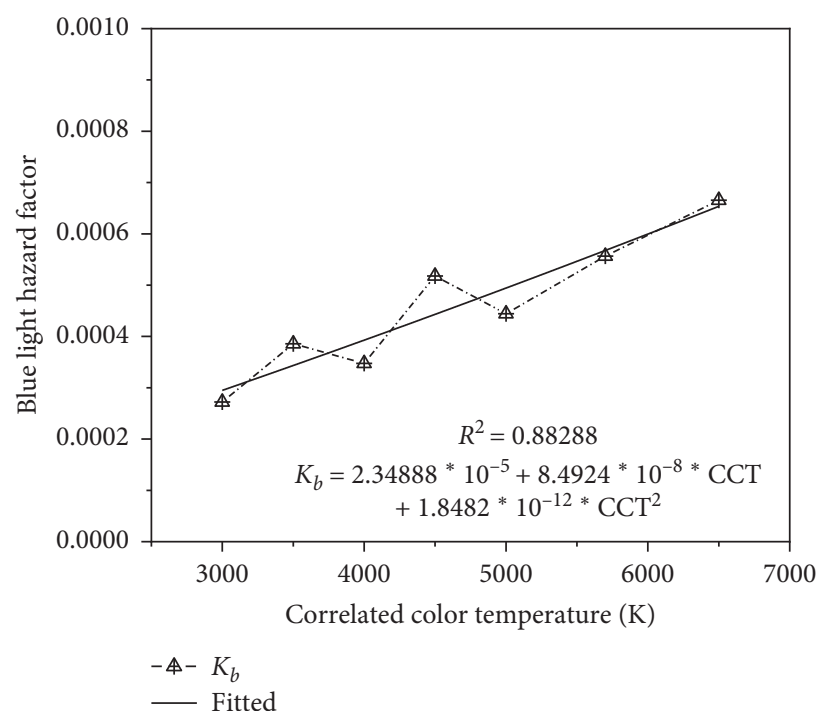

(c)

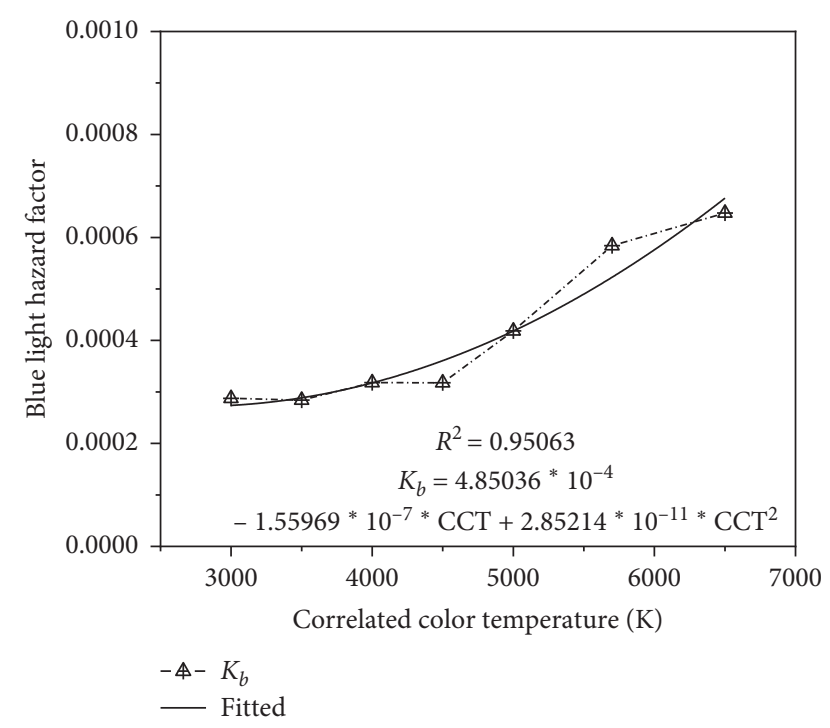

(b)

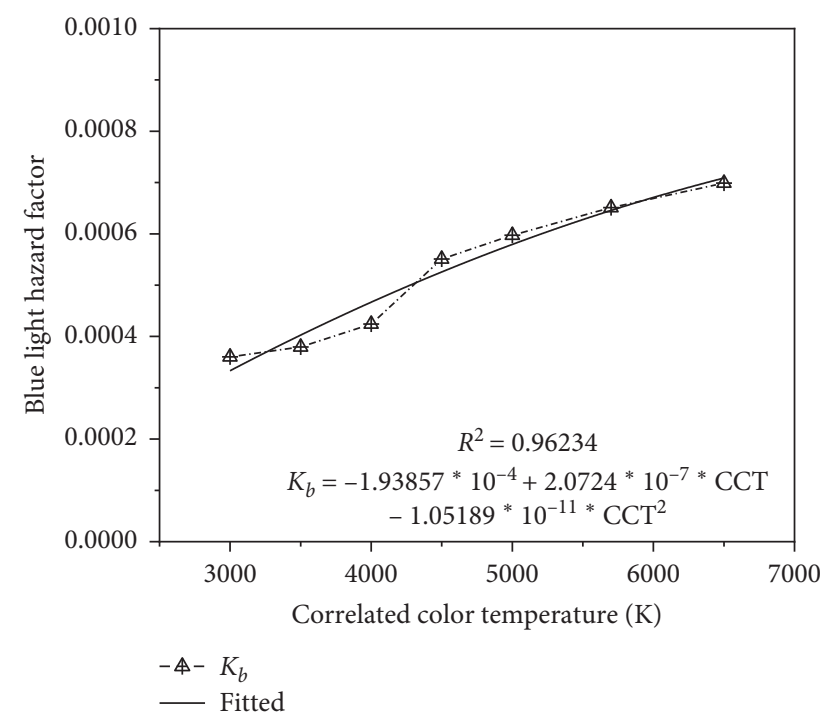

(d)

Figure 8: Blue light hazard factor. (a) CRI2012 $=65$. (b) CRI2012 $=75$. (c) CRI2012 $=85$. (d) CRI2012 $=95$.

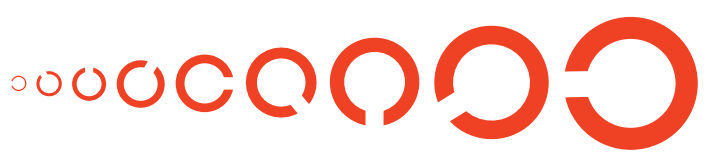

Figure 9: Observation targets.

TABLE 1: The corresponding target luminance to different background luminance.

\begin{tabular}{lc}
\hline Background luminance $\left(\mathrm{cd} / \mathrm{m}^{2}\right)$ & Target luminance $\left(\mathrm{cd} / \mathrm{m}^{2}\right)$ \\
\hline 1 & 1.4 \\
2 & 2.8 \\
3 & 4.2 \\
\hline
\end{tabular}

Table 2 shows the results of correlation and significance analysis with CFF drop value as target variables and CCT, CRI, luminance, and experimental duration as characteristic variables. In terms of correlation, CCT, CRI, and luminance are positively correlated with CFF drop value, while experimental duration is negatively correlated with CFF drop value. On the extent, experimental duration $>$ luminance $>$ CCT $>$ CRI, in which the experimental duration corresponds to high correlation, CCT and luminance correspond to low correlation, and CRI corresponds to weak correlation. This shows that the experimental duration has the highest impact on visual fatigue. Without changing the brightness of the tunnel light source, we can use the light source with high CCT and CRI characteristics 

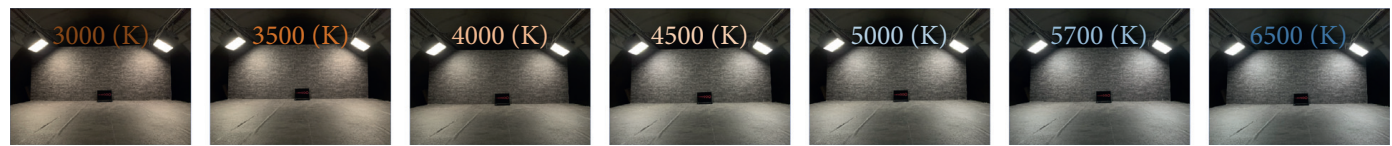

FIgURE 10: Experimental scene's layout.

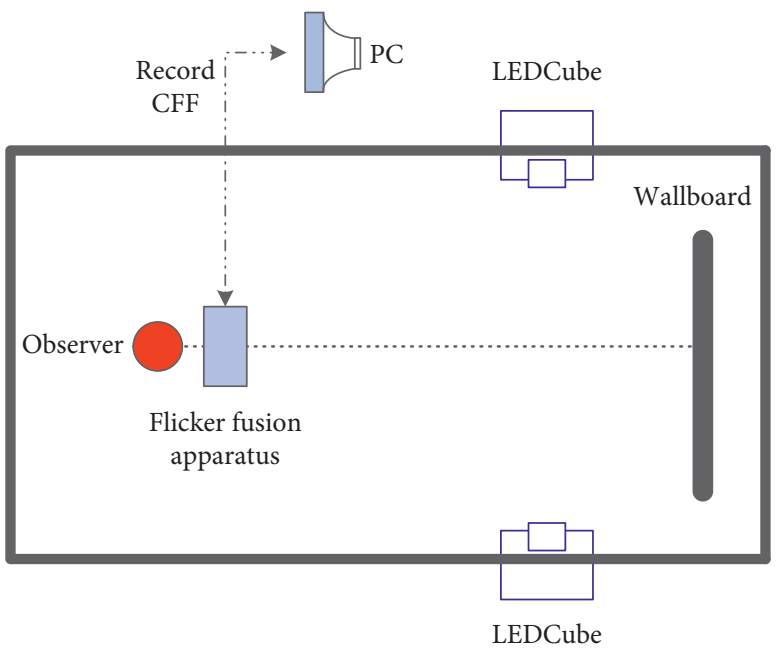

(a)

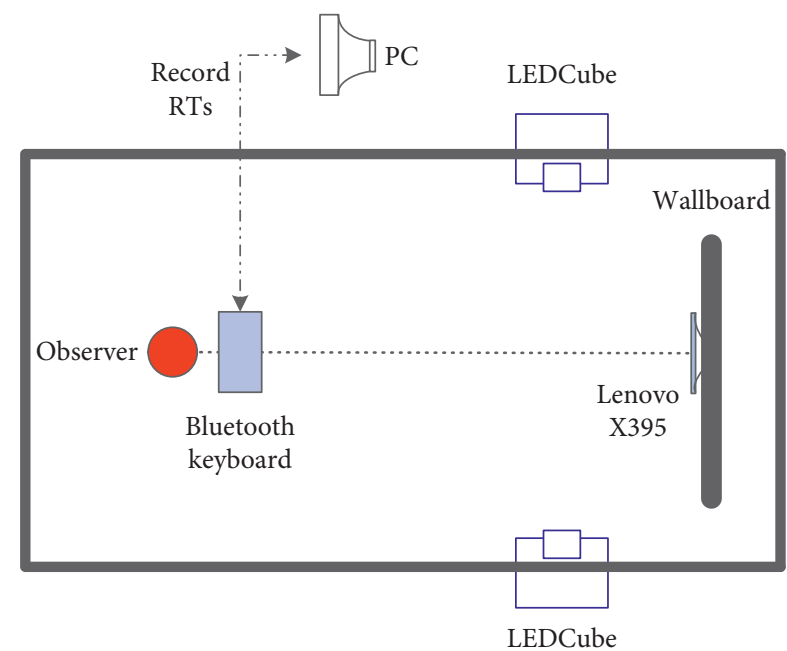

(b)

FIgURE 11: Schematic diagram of the experimental scene. (a) CFF. (b) Reaction time.

CCT (K)

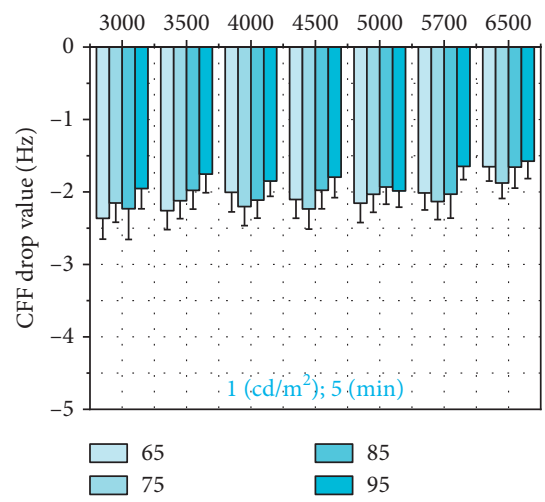

(a)

CCT (K)

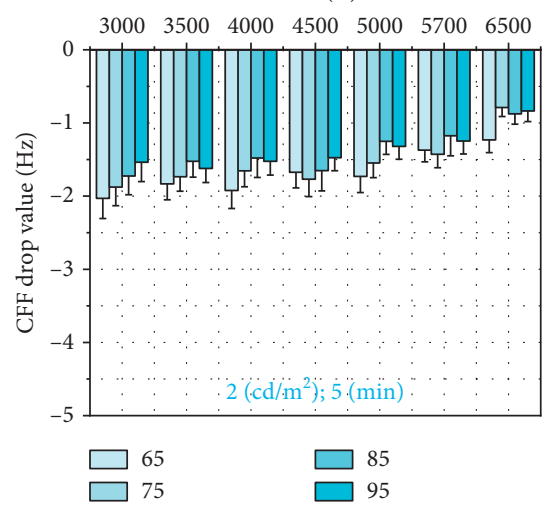

(d)

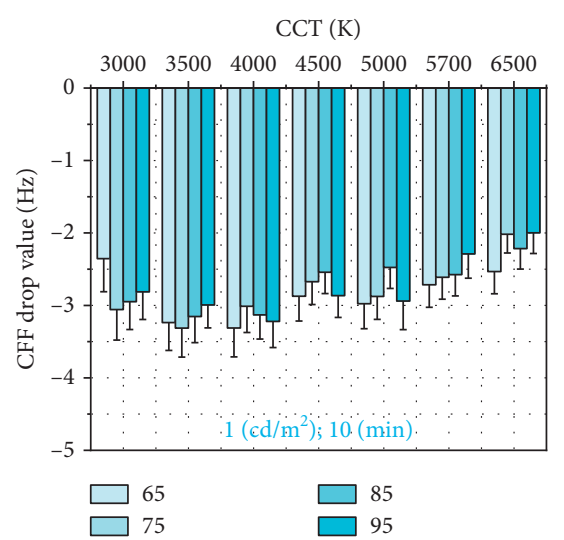

(b)

CCT (K)

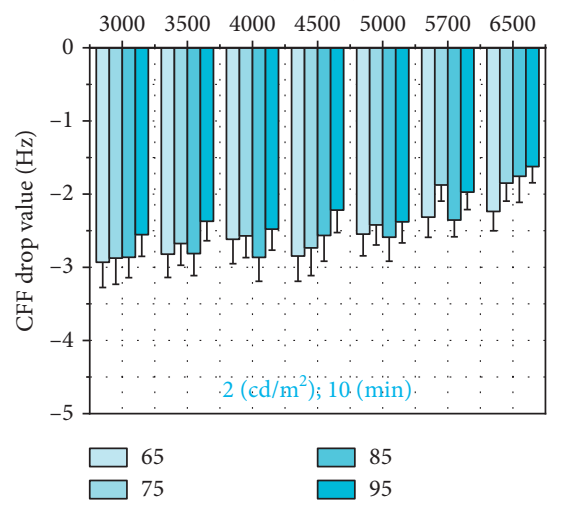

(e)

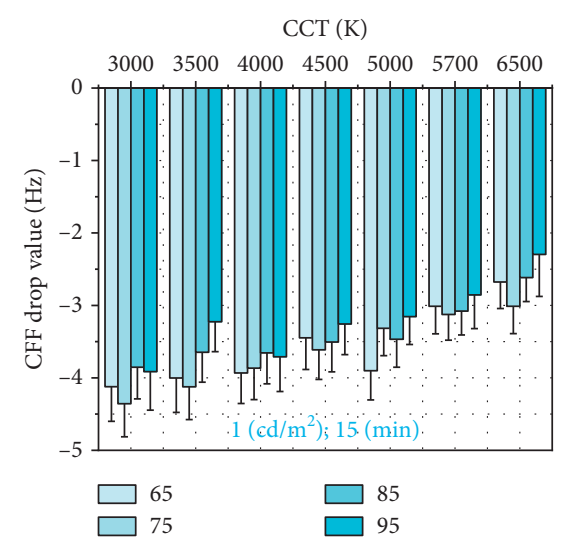

(c)

CCT $(\mathrm{K})$

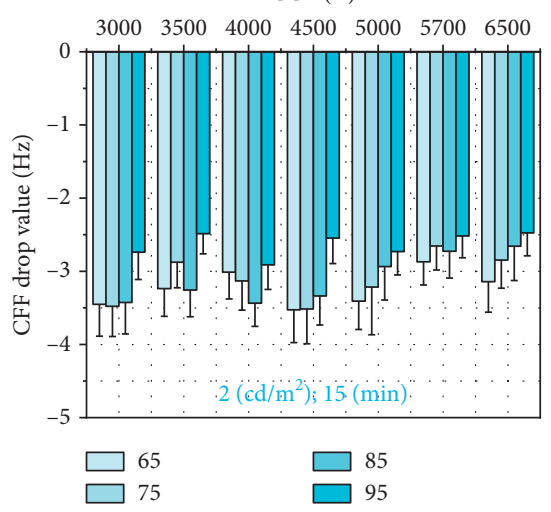

(f)

Figure 12: Continued. 
$\mathrm{CCT}(\mathrm{K})$

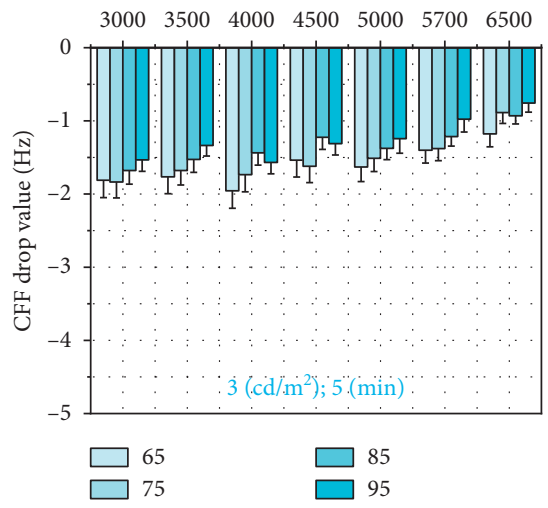

(g)
CCT (K)

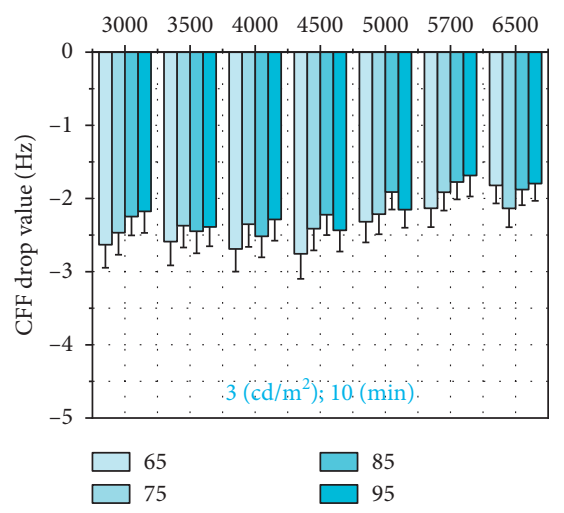

(h)

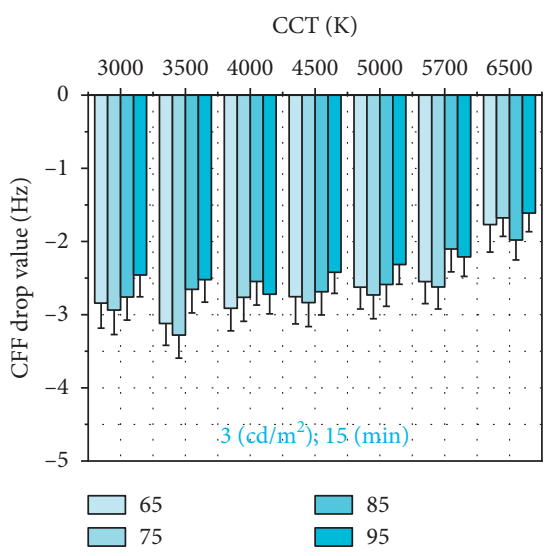

(i)

Figure 12: Average CFF drop value.

TABle 2: $P$ value of significance analysis for the above factors.

\begin{tabular}{lcccc}
\hline Parameters & CCT & CRI & Luminance & Experimental duration \\
\hline$r$ & $0.351^{* *}$ & $0.176^{* *}$ & $0.379^{* *}$ & $-0.770^{* *}$ \\
$P$ & $\leq 0.001$ & 0.005 & $\leq 0.001$ & $\leq 0.001$ \\
\hline
\end{tabular}

** Correlation is significant at the 0.01 level (2-tailed).

to alleviate the driver's visual fatigue to a certain extent. $P$ value showed that CCT, CRI, brightness, and experimental duration were significantly correlated at the level of 0.01 , which showed that the results were statistically significant.

4.2. Experimental Results and Discussion of Reaction Time. The average reaction time of 40 subjects in different light environments with time is shown in Figure 13.

Figure 13 shows the average reaction time of all observers in different light environments. It can be seen from Figure 13 that the reaction time increases with time, which indicates that the fatigue of the experimenter is increasing with time. When the luminance and CRI were fixed, the reaction time decreases with the increase of CCT. When the luminance and CCT were fixed, the reaction time decreases with the increase of CRI. When the CCT and CRI property were fixed, the reaction time decreases with the increase of luminance. This shows that when the tunnel lighting environment adopts high luminance, high CCT, and high CRI light source, the driver has a faster response when encountering dangerous situations, and the probability of traffic accidents will also be reduced. The experimental results are consistent with the above theoretical results. Table 3 shows the results of correlation and significance analysis of reaction time as target variables and CCT, CRI, luminance, and experimental duration as characteristic variables.

Table 3 shows the results of correlation and significance analysis of reaction time as target variables and CCT, CRI, luminance, and experimental duration as characteristic variables. In terms of correlation, CCT, CRI, and luminance are negatively correlated with reaction time, while experimental duration is positively correlated with reaction time.
On the extent, luminance $>$ experimental duration $>$ CCT $>$ CRI, in which the luminance corresponds to high correlation, experimental duration, CRI, and CCT correspond to low correlation. This shows that the experimental duration has the highest impact on fatigue. This means that to create a safe road environment, improving luminance is still the best choice. However, considering the tunnel energy saving, it is also a desirable method to improve the CCT and CRI of lamps.

4.3. Discussion on the Influence of the Spectrum on Visual and Nonvisual Effects. In order to study the correlation and significance between the above variables (CCT, CRI, Zvalue, perceived luminance, biological rhythm, blue light hazard, CFF decline value, and reaction time), in this paper, we use IBM SPSS software to do Pearson's correlation analysis on the experimental data, and the results are shown in Table 4.

From the table, we can see that compared with CCT and CRI, $Z$-value has a higher correlation with perceived brightness, biological rhythm, blue light hazard, and other parameters, reaching $0.939,0.974$, and 0.976 , respectively. This means that the content of blue light can better reflect the influence of the spectrum on biological effect. This means that future lighting standard design department can consider $Z$-value as one of the standards. From the perspective of blue light hazards, high CCT and CRI light sources have negative effects on visual health. However, it can improve the perceived brightness of human eyes, which are conducive to driving behavior under low illumination. At the same time, this study shows that the high CCT $(6500 \mathrm{~K})$ and high CRI (95) spectrum can improve the driver's visual performance 


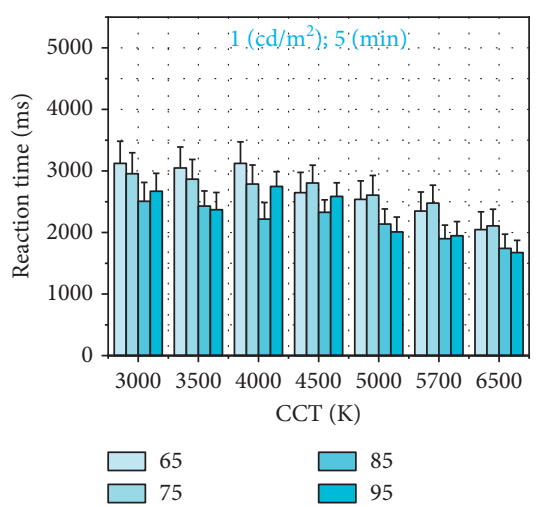

(a)

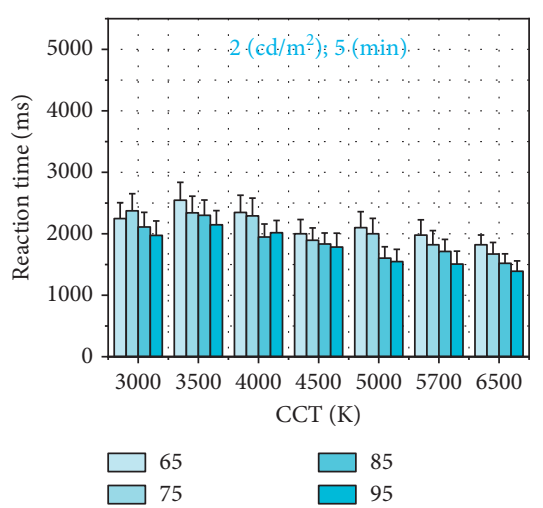

(d)

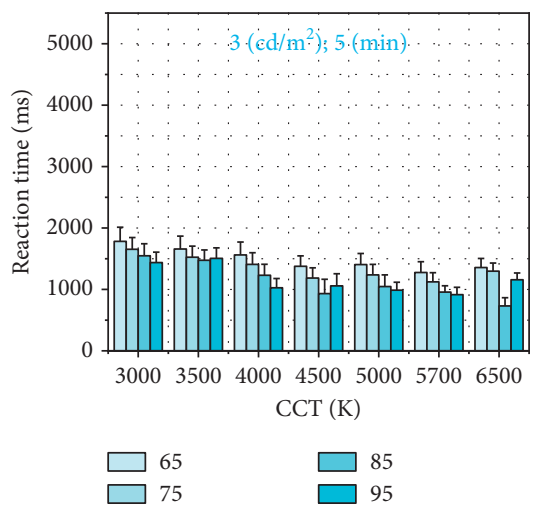

(g)
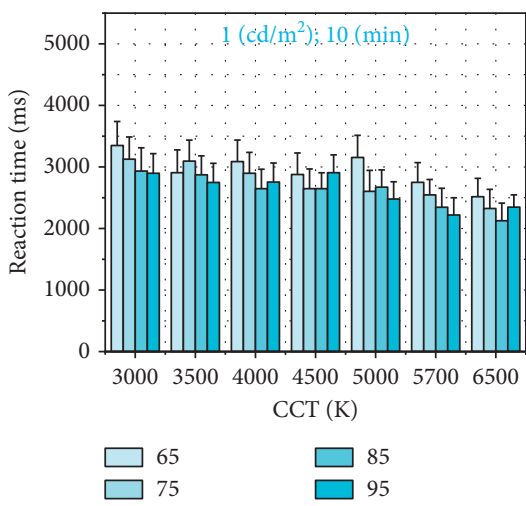

(b)

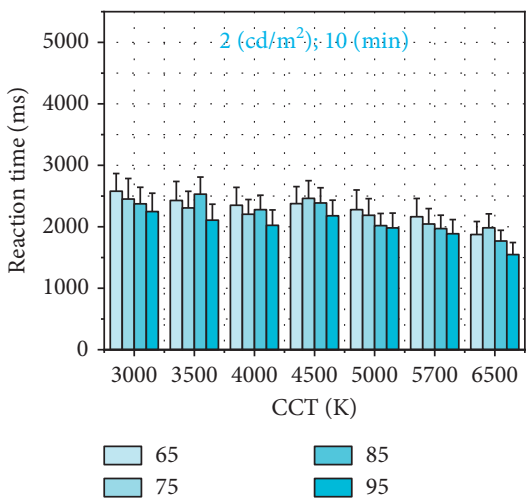

(e)

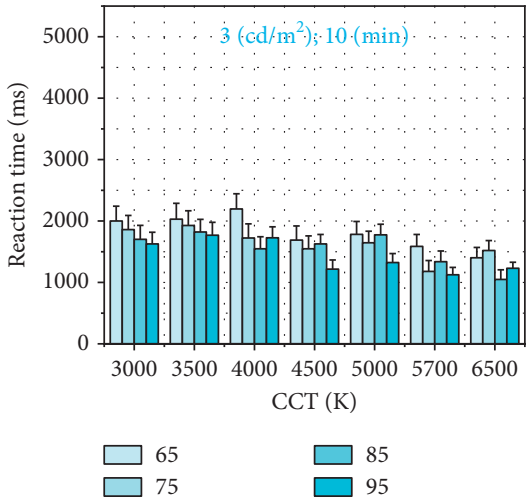

(h)
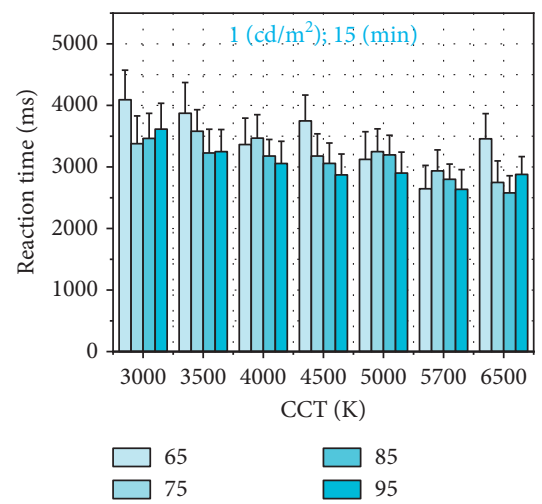

(c)
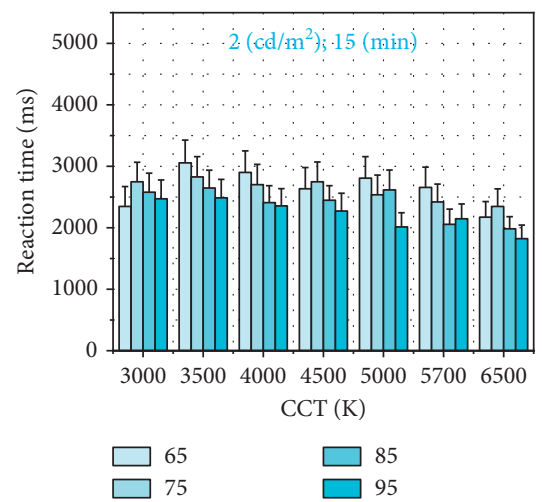

(f)

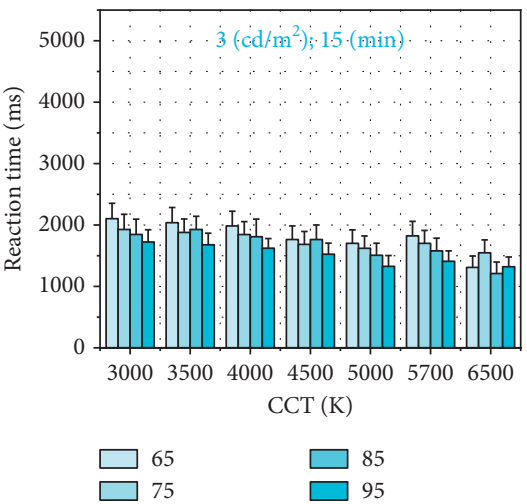

(i)

Figure 13: Average reaction time.

TABle 3: $P$ value of significance analysis for the above factors.

\begin{tabular}{lcccc}
\hline Parameters & CCT & CRI & Luminance & Experimental duration \\
\hline$r$ & $-0.335^{* *}$ & $-0.222^{* *}$ & $-0.804^{* *}$ & $0.350^{* *}$ \\
$P$ & 0.002 & 0.008 & $\leq 0.001$ & $\leq 0.001$ \\
\hline
\end{tabular}

${ }^{* *}$ Correlation is significant at the 0.01 level (2-tailed).

in the tunnel lighting environment with short lighting period. Current studies have shown that the damage and nonvisual biological effects of blue light are closely related to light intensity and lighting duration. Long time exposure to blue light can promote the visual center to make the ciliary muscle movement into a tight state, make people visual fatigue and blurred vision, and also affect melatonin secretion and circadian rhythm. This means that we can use high CCT $(6500 \mathrm{~K})$ and high CRI (95) light sources in the road lighting environment with short lighting period, and consider using low CCT and CRI light sources in other lighting environment with long lighting period. 
TABLE 4: Significance analysis for the above factors.

\begin{tabular}{|c|c|c|c|c|c|c|}
\hline & & CCT & CRI & $Z$-value & Visual luminance & Blue light hazard \\
\hline \multirow{2}{*}{ CRI } & $r$ & 0.000 & & & & \\
\hline & $P$ & 1.000 & & & & \\
\hline \multirow{2}{*}{$Z$-value } & $r$ & $0.877^{* *}$ & $0.418^{*}$ & & & \\
\hline & $P$ & $\leq 0.001$ & 0.027 & & & \\
\hline \multirow{2}{*}{ Visual luminance } & $r$ & $0.798^{* *}$ & $0.499^{* *}$ & $0.939^{* *}$ & & \\
\hline & $P$ & $\leq 0.001$ & 0.007 & $\leq 0.001$ & & \\
\hline \multirow{2}{*}{ Blue light hazard } & $r$ & $0.878^{* *}$ & 0.373 & $0.974^{* *}$ & $0.893^{* *}$ & \\
\hline & $P$ & $\leq 0.001$ & 0.051 & $\leq 0.001$ & $\leq 0.001$ & \\
\hline \multirow{2}{*}{ Circadian action factory } & $r$ & $0.874^{* *}$ & 0.370 & $0.976^{* *}$ & $0.880^{* *}$ & $0.983^{* *}$ \\
\hline & $P$ & $\leq 0.001$ & 0.053 & $\leq 0.001$ & $\leq 0.001$ & $\leq 0.001$ \\
\hline
\end{tabular}

${ }^{* *}$ Correlation is significant at the 0.01 level (2-tailed). ${ }^{*}$ Correlation is significant at the 0.05 level (2-tailed).

\section{Conclusions}

In this paper, the influence of spectrum on driver's visual and nonvisual biological effects under intermediate vision is studied. Firstly, combined with the influence of the spectrum on human visual and nonvisual biological effects, it is analyzed that the existing photometric test methods are not suitable for road traffic lighting and other intermediate visual brightness environment. Then, the intermediate visual spectral luminous efficiency curve is applied to the evaluation methods of nonvisual biological effects, perceived brightness, and blue light hazards. The results show that high CCT and CRI spectra with high blue light content can improve the perceived brightness, have stronger impact on human nonvisual effects, and cause more serious damage to blue light radiation. At the same time, the research shows that $Z$-value can better reflect the biological effect of the spectrum, and the lighting standard design department can consider $Z$-value as one of the standards in the future.

Then, a driving simulation experiment was carried out to test the driver's visual performance under different spectral illumination. Changes of CFF and RTS reflect the visual performance of drivers in different light environments. The results show that the light source with high CCT and high CRI corresponds to lower CFF drop value and RTS; that is to say, the driver's visual fatigue is lower, the visual effect is higher, and the driving behavior is more favorable under this light condition.

Through the study of the driving behavior of tunnel, it is found that the higher blue light content spectrum has a more positive effect on the visual efficiency when exposed to low light for a short time. Therefore, from the perspective of driving safety, this paper proposes to use LED with high CCT $(6500 \mathrm{~K})$ and high CRI (95) in the inner area of the tunnel to improve the driver's visual effect and driving safety. This paper provides a reasonable reference for tunnel lighting specification and tunnel lighting design department, which help to ensure personal safety and avoid traffic accidents inside the tunnel.

Although the current results are promising, they also have limitations. In the road environment with long lighting period, whether the LED light source with high CCT and CRI is harmful to driving behavior needs to be further studied. At the same time, for LED lighting, further experiments are needed to evaluate whether the circadian light value and blue light harm defined by the simplified model are consistent with the real situation. The current and previous studies have not fully clarified the physiological mechanism related to these results, and further research is needed.

\section{Data Availability}

The data used to support the findings of this study are available from the corresponding author upon request.

\section{Conflicts of Interest}

The authors declare that there are no conflicts of interest regarding the publication of this paper.

\section{Acknowledgments}

This study was supported in part by the National Natural Science Foundation of China under Grant no. 61701069 and in part by the Fundamental Research Funds for the Central Universities of China under Grants nos. 3132019340 and 3132019200 .

\section{References}

[1] L. Bier, P. Wolf, H. Hilsenbek, and B. Abendroth, "How to measure monotony-related fatigue? A systematic review of fatigue measurement methods for use on driving tests," Theoretical Issues in Ergonomics Science, vol. 21, no. 1, pp. 22-55, 2018.

[2] T. Vogelpohl, M. Kühn, T. Hummel, and M. Vollrath, “Asleep at the automated wheel-Sleepiness and fatigue during highly automated driving," Accident Analysis \& Prevention, vol. 126, pp. 70-84, 2019.

[3] A. Chaudhuri and A. Routray, "Driver fatigue detection through chaotic entropy analysis of cortical sources obtained from scalp EEG signals," IEEE Transactions on Intelligent Transportation Systems, vol. 21, no. 1, pp. 185-198, 2020.

[4] S. Bassan, "Overview of traffic safety aspects and design in road tunnels,” IATSS Research, vol. 40, no. 1, pp. 35-46, 2016. 
[5] R. Li, Y. V. Chen, and L. Zhang, "Effect of music tempo on long-distance driving: which tempo is the most effective at reducing fatigue?" i-Perception, vol. 10, no. 4, 2019.

[6] M. C. Zheng, "The design of wall pictures to relieve driving fatigue in the long tunnel," in Proceedings of the International Conference on Cooperative Design, Visualization and Engineering, Mallorca, Spain, 2015.

[7] H. Huang, Y. Peng, J. Wang, Q. Luo, and X. Li, "Interactive risk analysis on crash injury severity at a mountainous freeway with tunnel groups in China," Accident Analysis \& Prevention, vol. 111, pp. 56-62, 2018.

[8] A. Pervez, H. Huang, J. Lee, C. Han, and X. Zhang, "Crash analysis of expressway long tunnels using a seven-zone analytic approach," Journal of Transportation Safety \& Security, vol. 13, pp. 108-122, 2019.

[9] Y. Ying, X. Wang, L. Shi, and H. Liu, "Influence of light zones on drivers' visual fixation characteristics and traffic safety in extra-long tunnels," Traffic Injury Prevention, vol. 18, pp. 102-110, 2016.

[10] R.-H. Huang, L. Lee, Y. Chu, and S. Yi, "Effects of correlated color temperature on focused and sustained attention under white LED desk lighting," Color Research \& Application, vol. 40, pp. 281-286, 2014.

[11] People's Communications Press, Highway Tunnel Lighting Design Rules: JTG/T D70/2-01-2014, People's Communications Press, Beijing, China, 2014.

[12] M. G. Figueiro, A. Bierman, B. Plitnick, and M. S. Rea, "Preliminary evidence that both blue and red light can induce alertness at night," BMC Neuroscience, vol. 10, no. 1, p. 105, 2009.

[13] M. S. Rea, A. Bierman, M. G. Figueiro, and J. D. Bullough, “A new approach to understanding the impact of circadian disruption on human health," Journal of Circadian Rhythms, vol. 6, p. 7, 2008.

[14] J. H. Oh, H. Yoo, H. K. Park, and Y. R. Do, "Analysis of circadian properties and healthy levels of blue light from smartphones at night," Scientific Reports, vol. 5, no. 1, Article ID 11325, 2015.

[15] F. Rao, A. H. S. Chan, and X.-f. Zhu, "Effects of photopic and cirtopic illumination on steady state pupil sizes," Vision Research, vol. 137, pp. 24-28, 2017.

[16] C. Christian, M. Mirjam, K. Szymon et al., "High sensitivity of human melatonin, alertness, thermoregulation, and heart rate to short wavelength light," Journal of Clinical Endocrinology and Metabolism, vol. 90, pp. 1311-1316, 2005.

[17] J. Phipps-Nelson, J. R. Redman, L. J. M. Schlangen, and S. M. W. Rajaratnam, "Blue light exposure reduces objective measures of sleepiness during prolonged nighttime performance testing," Chronobiology International, vol. 26, pp. 891-912, 2009.

[18] L. Sahin and M. G. Figueiro, "Alerting effects of shortwavelength (blue) and long-wavelength (red) lights in the afternoon," Physiology \& Behavior, vol. 116-117, pp. 1-7, 2013.

[19] N. I. Lisdiani and Yassierli, "Blue light exposure improves awareness during monotonous activities at night," in Proceedings of the 2012 Southeast Asian Network of Ergonomics Societies Conference (SEANES), Langkawi, Malaysia, 2012.

[20] M. G. Figueiro, L. Sahin, B. Wood, and B. Plitnick, "Light at night and measures of alertness and performance: implications for shift workers," Biological Research for Nursing, vol. 18, no. 1, pp. 90-100, 2015.

[21] L. Molcan, H. Sutovska, M. Okuliarova, T. Senko, L. Krskova, and M. Zeman, "Dim light at night attenuates circadian rhythms in the cardiovascular system and suppresses melatonin in rats," Life Sciences, vol. 231, Article ID 116568, 2019.

[22] S. M. Berman, "A new retinal photoreceptor should affect lighting practice," Lighting Research \& Technology, vol. 40, no. 4, pp. 373-376, 2008.

[23] L.-L. Zheng, T.-Z. Wu, Y.-J. Lu et al., "Spectral optimization of three-primary LEDs by considering the circadian action factor," IEEE Photonics Journal, vol. 8, no. 6, 2016.

[24] L. Bellia and F. Bisegna, "From radiometry to circadian photometry: a theoretical approach," Building and Environment, vol. 62, pp. 63-68, 2013.

[25] T. Wu, Y. Lin, H. Zhu et al., "Multi-function indoor light sources based on light-emitting diodes-a solution for healthy lighting," Optics Express, vol. 24, no. 21, pp. 24401-24412, 2016.

[26] D. Gall and K. Bieske, Definition and Measurement of Circadian Radiometric Quantities, Technical University of Ilmenau, Ilmenau, Germany, 2004.

[27] L. Dong, L. Qin, W. Xu, and L. Zhang, "The impact of LED correlated color temperature on visual performance under mesopic conditions," IEEE Photonics Journal, vol. 9, no. 6, 2017.

[28] G. C. Brainard, J. P. Hanifin, J. M. Greeson et al., "Action spectrum for melatonin regulation in humans: evidence for a novel circadian photoreceptor," The Journal of Neuroscience, vol. 21, pp. 6405-6412, 2001.

[29] J. Ju, "The non-visual biological effect of lighting and its practical meaning," China Illuminating Engineering Journal, vol. 20, pp. 25-28, 2009.

[30] K. Thapan, J. Arendt, and D. J. Skene, "An action spectrum for melatonin suppression: evidence for a novel non-rod, noncone photoreceptor system in humans," Journal of Physiology, vol. 535, pp. 261-267, 2010.

[31] S. A. Rahman, M. Hilaire, and S. W. Lockley, "The effects of spectral tuning of evening ambient light on melatonin suppression, alertness and sleep," Physiology \& Behavior, vol. 177, 2017.

[32] R. Nagare, M. S. Rea, B. Plitnick, and M. G. Figueiro, "Nocturnal melatonin suppression by adolescents and adults for different levels, spectra, and durations of light exposure," Journal of Biological Rhythms, vol. 34, no. 2, pp. 178-194, 2019.

[33] R. Nagare, B. Plitnick, and M. G. Figueiro, "Effect of exposure duration and light spectra on nighttime melatonin suppression in adolescents and adults," Lighting Research and Technology, vol. 51, 2018.

[34] A. S. Prayag, R. P. Najjar, and C. Gronfier, "Melatonin suppression is exquisitely sensitive to light and primarily driven by melanopsin in humans," Journal of Pineal Research, vol. 66, Article ID e12562, 2019.

[35] S. Lee, K. Matsumori, K. Nishimura et al., "Melatonin suppression and sleepiness in children exposed to blue-enriched white LED lighting at night," Physiological Reports, vol. 6, Article ID e13942, 2018.

[36] D. Gall, "Circadiane lichtgrößen und deren messtechnische ermittlung," Licht, vol. 54, pp. 1292-1297, 2002.

[37] R. L. Tomkiewicz and W. Cohen, "The effects of changes in arousal level on critical flicker fusion frequency and figural reversal tasks," Psychophysiology, vol. 6, pp. 421-428, 2010.

[38] J. Formanek, R. Fischer, and D. Frantikova, "The critical flicker fusion frequency and the frequency of neurotic manifestations in various occupations," Activitas Nervosa Superior, vol. 4, p. 183, 1962.

[39] A. Eisen-Enosh, N. Farah, Z. Burgansky-Eliash, U. Polat, and Y. Mandel, "Evaluation of critical flicker-fusion frequency 
measurement methods for the investigation of visual temporal resolution,” Scientific Reports, vol. 7, no. 1, p. 15621, 2017.

[40] R. H. Peckham and W. M. Hart, "Critical flicker frequency, photochemical mechanisms, and perceptual responses," Archives of Ophthalmology, vol. 60, no. 3, pp. 461-471, 1958.

[41] S. Park, G. Kyung, D. Choi et al., "Effects of display curvature and task duration on proofreading performance, visual discomfort, visual fatigue, mental workload, and user satisfaction," Applied Ergonomics, vol. 78, pp. 26-36, 2019.

[42] C. W. Lin, F. M. Yeh, B. W. Wu, and C. H. Yang, "The effects of reflected glare and visual field lighting on computer vision syndrome," Clinical and Experimental Optometry, vol. 102, pp. 513-520, 2019.

[43] Y. Ikushima, H. Yabuuchi, J. Morishita, and H. Honda, "Analysis of dominant factors affecting fatigue caused by softcopy reading," Academic Radiology, vol. 20, no. 11, pp. 1448-1456, 2013.

[44] C.-J. Chao, Y.-J. Yau, C.-H. Lin, and W.-Y. Feng, "Effects of display technologies on operation performances and visual fatigue," Displays, vol. 57, pp. 34-46, 2019.

[45] B. Liang, S. He, L. Tähkämö et al., "Lighting for road tunnels: the influence of CCT of light sources on reaction time," Displays, vol. 61, Article ID 101931, 2020.

[46] Y. Yang, C. Z. Zhu, and L. Wang, "Highway tunnel lighting research based on mesopic vision," Applied Mechanics and Materials, vol. 71-78, pp. 4061-4064, 2011.

[47] N. Arbabzadeh, M. Jafari, M. Jalayer, S. Jiang, and M. Kharbeche, "A hybrid approach for identifying factors affecting driver reaction time using naturalistic driving data," Transportation Research Part C: Emerging Technologies, vol. 100, pp. 107-124, 2019.

[48] L. B. Lempke, R. C. Lynall, N. L. Hoffman, H. Devos, and J. D. Schmidt, "Slowed driving-reaction time following concussion-symptom resolution," Journal of Sport and Health Science, vol. 10, no. 2, pp. 145-153, 2021.

[49] S. Y. He, L. Tähkämö, M. Maksimainen, B. Liang, G. B. Pan, and L. Halonen, "Effects of transient adaptation on drivers' visual performance in road tunnel lighting," Tunnelling and Underground Space Technology, vol. 70, pp. 42-54, 2017. 